\title{
Disk and wind evolution of Achernar: the breaking of the fellowship
}

\author{
S. Kanaan ${ }^{1}$, A. Meilland ${ }^{1}$, Ph. Stee ${ }^{1}$, J. Zorec ${ }^{3}$, A. Domiciano de Souza ${ }^{2}$, Y. Frémat ${ }^{4}$, and D. Briot ${ }^{5}$ \\ 1 UMR 6525 CNRS H. FIZEAU UNS, OCA, Campus Valrose, 06108 Nice Cedex 2; CNRS - Avenue Copernic, Grasse, France \\ e-mail: Samer . Kanaan@obs-azur. fr \\ 2 UMR 6525 CNRS H. FIZEAU UNS, OCA, Campus Valrose, 06108 Nice Cedex 2, France \\ 3 Institut d'Astrophysique de Paris, UMR7095 CNRS, Univ. P. \& M. Curie, 98bis Bd. Arago, 75014 Paris, France \\ ${ }^{4}$ Royal Observatory of Belgium, 3 Av. circulaire, 1180 Bruxelles, Belgium \\ 5 Observatoire de Paris, 61 Avenue de l'Observatoire, 75014 Paris, France
}

Received 18 October 2007 / Accepted 7 April 2008

\section{ABSTRACT}

\begin{abstract}
Aims. We use spectral energy distributions (SEDs), $\mathrm{H} \alpha$ line profiles, and visibilities available in the literature to study Achernar's envelope geometry and to propose a possible scenario for its circumstellar disk formation and dissipation.

Methods. We use the SIMECA code to investigate possible geometries of the circumstellar environment by comparing our synthetic results with spectroscopic and high angular resolution data from the VLTI/VINCI instrument. We compute three different kinds of models: an equatorial disk, a polar wind, and a disk+wind model.

Results. We develop a 2D axial symmetric kinematic model to study the variation of the observed $\mathrm{H} \alpha$ line profiles, which provides clear evidence of Achernar's equatorial disk formation and dissipation between 1991 and 2002. Our model can reproduce the polarwind extension greater than $10 R_{\star}$ and a possible equatorial disk $\left(\leq 5 R_{\star}\right)$ but we were unable to estimate the wind opening angle. We reproduce the $\mathrm{H} \alpha$ line-profile variations using an outburst scenario, but the disk final contraction requieres an additional physical effect to be taken into account. The polar stellar wind does not appear to be linked to the presence of a disk or a ring around the star. We test the possibility of a binary companion to Achernar, as found by Kervella \& Domiciano de Souza (2007, A\&A, 474, L49), but conclude that the VLTI/VINCI visibilities cannot be explained by a rotationally-distorted Be star and a companion alone. The presence of a polar jet provides an important component to reproduce the observations even if it is not excluded that the companion could partially influence the observations. New interferometric observations at short baselines $(5 \leq B \leq 20 \mathrm{~m})$ are mandatory to constrain Achernar's circumstellar envelope, as well as spectroscopic long-term follow-up observational programs to link Achernar's mass-loss episodes with its circumstellar disk formation.
\end{abstract}

Key words. techniques: high angular resolution - techniques: interferometric - stars: emission-line, Be - stars: winds, outflows stars: individual: Achernar - stars: circumstellar matter

\section{Introduction}

Observations of Be stars provide clear evidence that these stars are variable with a timescale of variability that ranges between minutes and decades (Porter \& Rivinius 2003). Phase transitions are revealed by Balmer line-emission intensity and global lineprofile changes as well as changes in the visible spectral energy distribution. Variations in the emission lines can occur over periods of years, months, or even days (Porter \& Rivinius 2003). These changes can be due either to variations in the physical structure and size of a more or less permanent circumstellar environment $(\mathrm{CE})$, or the creation of a new $\mathrm{CE}$ during mass ejection events of the central star. Interferometric and linear-polarization measurements place observational constraints on the CE flattening (Gies et al. 1990; Stee et al. 1994; Quirrenbach et al. 1997; Yudin 2001; Tycner 2004). Nevertheless, the disk formation and its geometry is still poorly known, but the situation is changing rapidly with the recent CHARA (Gies et al. 2007), NPOI (Tycner et al. 2006), and VLTI interferometric measurements (Meilland et al. 2007).

Studying the effects of the gravitational darkening on the measure of the projected rotational-velocity $V \sin i$ parameters, Stoeckley (1968) suggested that Be stars could be critical rotators, so that "...mild prominence activity or other minor disturbances lead to the ejection of matter...". These arguments were further developed by Townsend et al. (2004) and Owocki (2004). By determining the fundamental parameters of $130 \mathrm{Be}$ stars and taking account of gravitational darkening, Fremat et al. (2005) showed that Be stars rotate, on average, with an angular velocity ratio $\omega=\Omega / \Omega_{\mathrm{c}} \approx 0.88$. On the other hand, interferometric measurements of the brightest known Be star, $\alpha$ Eri (Achernar), carried out by Domiciano de Souza et al. (2003), suggest that this star has a flattening that exceeds the predictions for a critical rigid rotator Roche model.

Our first objective is to develop a physical model of a Achernar circumstellar envelope that describes the entire VLTI/ VINCI data set acquired by Kervella \& Domiciano de Souza (2006, hereafter KD). This will help determine whether the flattening ratio is smaller than the value estimated by $\mathrm{KD}$, or whether it can be accounted for by a small circumstellar contribution. The second objective is to compare our results with the finding of a circumstellar environment around Achernar by Vinicius et al. (2006) and study its possible disk evolution between 1991 and 2002.

The paper contains the following main sections:

- Section 3: we introduce the SIMECA code used to interpret Achernar photometric, spectroscopic, and interferometric data, and describe and explain the modifications that we make to model this particular star. 
- Section 4: we explore possible scenarios based on disk and/or wind formation and attempt to achieve the most appropriate matches between the data and our synthetic observables, namely the SED, the visibilities, and the line profiles.

- Section 5: we discuss the possible formation/dissipation processes of Achernar's circumstellar envelope.

- In the last section, we summarize our results and conclusions.

\section{Achernar}

The star Achernar ( $\alpha$ Eridani, HD 10144) is the nearest $(d=$ $44 \mathrm{pc}$, Hipparcos) and brightest $(V=0.46 \mathrm{mag}) \mathrm{Be}$ star. Depending on the author and technique used, the spectral type of Achernar ranges from B3-B4IIIe to B4Ve (e.g. Slettebak 1982; Balona et al. 1987).

The measured projected rotational velocity $V \sin i$ ranges from 220 to $270 \mathrm{~km} \mathrm{~s}^{-1}$, and the effective temperature $T_{\text {eff }}$ from 15000 to $20000 \mathrm{~K}$ (e.g., Rivinius, priv. comm.; Chauville et al. 2001; Vinicius et al. 2006). Achernar's rapid rotation, witch corresponds to $\geq 80 \%$ of its critical velocity, has two significant effects on stellar structure: a rotational flattening and a gravity darkening described by the von Zeipel effect (Von Zeipel 1924).

Achernar's rotational flattening was measured for the first time by Domiciano de Souza et al. (2003), using the VLTI and its test-bed VINCI instrument. The measured flattening ratio was $R_{\text {eq }} / R_{\text {pole }} \gtrsim 1.5$.

By analysing the $\mathrm{H} \alpha$ line-profile variations, Vinicius et al. (2006) proposed that a circumstellar disk was present, as opposed to a completely-flattened photosphere that had no circumstellar contribution (see Figs. 10 and 11). However, KD reprocessed the entire VINCI visibility data set, which had an almost complete $(u, v)$ plane coverage to ascertain whether there was either an equatorial or a polar, gaseous extension, or both. They concluded that there might be a polar extension.

\section{SIMECA: a code dedicated to active hot stars}

The SIMECA code calculates photometric and spectroscopic measurements. In addition, it derives both hydrogen-line and continuum intensity maps to obtain theoretical visibility curves that can be directly compared to high angular-resolution data. The main hypothesis of this code is that the envelope is axisymmetric with respect to the rotational axis. It is assumed that the physics of the polar regions is represented by a CAK-type stellar-wind model (Castor et al. 1975), while, for other stellar latitudes, a parameterized model, constrained by spectrallyresolved interferometric data, is adopted. We use a polar, terminal velocity of $1000 \mathrm{~km} \mathrm{~s}^{-1}$, which may be larger than predicted by the bi-stability theory of Lamers \& Pauldrach (1991) of about 1.3 times the escape velocity, i.e. $725 \mathrm{~km} \mathrm{~s}^{-1}$. This small difference in the polar, terminal velocity, however, has no influence on the following results, in particular the polar extension determined using SIMECA. The inner equatorial region is dominated by Keplerian rotation.

The ionization-excitation equations of hydrogen were solved for an envelope that was modeled as a $411 \times 92 \times 73$ cube using spherical coordinates $(r, \theta$ and $\phi)$ and assuming a Planck function for the radiation field emitted by the central star. Since the final population of atomic levels are strongly-NLTE distributed, we start with the LTE populations for each level, compute the escape probability of each transition, which enables updated populations to be determined, and then iterate until convergence.
The convergence is quite rapid, occuring within about ten iterations, and stable for effective temperatures of the central star in the range $10000<T_{\text {eff }}<40000$. The basic equations of the SIMECA code were given in detail by Stee \& Araújo (1994).

To account for the photospheric, absorption-line, we assumed that the underlying star was a normal B star with a specific $\left(T_{\text {eff }}, \log g\right)$ parameters. For these parameters, we then compute the Br $\gamma$ synthetic line-profile, using the SYNSPEC code developed by Hubeny (Hubeny 1988; Hubeny \& Lanz 1995), where the gravitational-darkening effect (von Zeipel 1924) was not included. These photospheric, line-profiles were then broadened by solid rotation, and possibly absorbed further by the envelope volume, projected on to the stellar disk. Since kinematics was the dominant effect on the line-profile shape, neglecting the von Zeipel effect in the computation of the photospheric, linecomponent, was not crucial and did not significantly influence the main conclusions presented in the remainder of this paper. We note that the SIMECA code is also able to produce theoretical, intensity maps of the circumstellar envelope for both this line and the continuum at different wavelengths and within a given spectral bandwith, for instance the $K$ band used for the VINCI measurments. These maps can be directly compared to the milli-arcsecond, interferometric measurements, such as those obtained from the VLTI interferometer.

The variation of the mass flux $(\phi)$, from the pole to the equator, is given by (see Stee 2003, for more details):

$\phi(\theta)=\phi_{\text {pole }}+\left[\left(\phi_{\text {eq. }}-\phi_{\text {pole }}\right) \sin ^{m 1}(\theta)\right]$

where $\theta$ is the stellar colatitude. A small $m 1$ value $(m 1 \lesssim 0.1)$ corresponds to a nearly spherical outflow whereas a large one $(m 1 \gtrsim 10)$ mimics outflow into a very thin disk. Since the contribution of the polar, stellar wind is week and the terminal velocity in the dense, equatorial region is only $10 \mathrm{~km} \mathrm{~s}^{-1}$, the disk is almost Keplerian. We assume that $V \sin i=230 \mathrm{~km} \mathrm{~s}^{-1}$, even if Vinicius et al. (2006) found $V \sin i=242 \pm 16 \mathrm{~km} \mathrm{~s}^{-1}$, after correcting for uncertainties induced by the gravitational-darkening effect and assuming that $\omega=\Omega / \Omega_{\mathrm{c}} \approx 0.79$. Since the star may not follow a rigid rotation, its true $V \sin i$ may, however, be closer to its apparent value, which would not strongly modify the local values of the gravity and effective temperature as found when assuming solid-body rotation. Thus, we obtain a rotational velocity of $V_{K}=304 \mathrm{~km} \mathrm{~s}^{-1}$, at the stellar surface, using $i=$ $50^{\circ}$ (Domiciano de Souza et al. 2003). This produces a typical double-peaked, emission, line-profile, with intensity maps that depend strongly on the observed wavelength.

The continuum emission is computed by taking into account free-free and free-bound emissions, and the absorption coefficient free-free and scattering mechanisms. The free-free emission coefficient is given by:

$\eta_{\mathrm{ff}}=5.441 \times 10^{-39} n_{\mathrm{e}}^{2} T^{-1 / 2} \mathrm{e}^{\frac{-h \nu}{k T}}$

the free-bound emission coefficient for $v \gtrsim v_{\mathrm{ci}}\left(v_{\mathrm{ci}}\right.$ is the $i$ th series limit frequency) is

$\eta_{\mathrm{fb}}=\sum_{n} 2.14 \times 10^{-32} n_{\mathrm{e}}^{2} i^{-3} T^{-3 / 2} \mathrm{e}^{\frac{h\left(v_{\mathrm{c}}-v\right)}{k T}}$

the free-free absorption coefficient is:

$\kappa_{\mathrm{ff}}=3.69 \times 10^{8} n_{\mathrm{e}}^{2} T^{-1 / 2} v^{-3}\left(1-\mathrm{e}^{\frac{-h \nu}{k T}}\right)$

and the scattering coefficient is $n_{\mathrm{e}} \sigma$. The basic parameters used in the present paper to simulate the Be-star circumstellar-disk are given in Table 1. 
Table 1. Parameters and results obtained in this paper to simulate Achernar's circumstellar envelope.

\begin{tabular}{c|c}
\hline \hline$T_{\text {eff }}($ eq) & $8500 \mathrm{~K}$ \\
$T_{\text {eff }}$ (pole) & $20000 \mathrm{~K}$ \\
Radius (eq) & $11.8 R_{\odot}$ \\
Radius (pole) & $7.6 R_{\odot}$ \\
Inclination angle $i$ & $50^{\circ}$ \\
Photospheric density & $1.0 \times 10^{-12} \mathrm{~g} \mathrm{~cm}^{-3}$ \\
Photospheric expansion velocity & $1.00 \mathrm{~km} \mathrm{~s}^{-1}$ \\
Equatorial rotation velocity & $304 \mathrm{~km} \mathrm{~s}^{-1}$ \\
Equatorial terminal velocity & $10 \mathrm{~km} \mathrm{~s}^{-1}$ \\
Polar terminal velocity & $1000 \mathrm{~km} \mathrm{~s}^{-1}$ \\
Polar mass flux & $4.0 \times 10^{-12} M_{\odot} \mathrm{year}^{-1} \mathrm{sr}^{-1}$ \\
Equatorial mass flux $\left(M_{\odot}\right.$ year & $\mathrm{sr}^{-1}$ ) \\
$m 1$ & $4.0 \times 10^{-14}<\phi_{\mathrm{eq}}<4.0 \times 10^{-12}$ \\
Mass of the envelope & 0.01 \\
Mass loss & $0.742 \times 10^{-11} M_{\odot}$ \\
\hline
\end{tabular}

Or the apparent stellar shape, we adopt stellar parameter values measured directly from interferometry (see $\mathrm{KD}$ ): $R_{\mathrm{eq}} / R_{\text {pole }}=$ 1.41 , with $R_{\text {pole }}=7.1 R_{\odot}$. Since the original SIMECA code assumes spherical stars with uniform $T_{\text {eff }}$ over the entire surface, we introduced an elliptical star, according to the following representation:

$\left[\frac{R(\theta)}{R_{\text {eq }}}\right]_{\text {proj }}=\left(\frac{R_{\text {pole }}}{R_{\text {eq }}}\right)_{\text {phys }}\left\{1-\left[1-\left(\frac{R_{\text {pole }}}{R_{\text {eq }}}\right)_{\text {phys }}^{2}\right] \sin ^{2} \theta\right\}^{1 / 2}$

where $\theta$ is the stellar colatitude. We note, however, that the physical or true ratio of the stellar radii $\left(R_{\text {pole }} / R_{\text {eq }}\right)_{\text {phys }}$, given by an approximation similar to Eq. (5), which relates the apparent and projected ratios by:

$$
\left[\frac{R_{\mathrm{eq}}}{R_{\mathrm{pole}}}\right]_{\text {proj }}=\left\{1+\left[\left(\frac{R_{\mathrm{eq}}}{R_{\mathrm{pole}}}\right)_{\text {phys }}^{2}-1\right] \sin ^{2} i\right\}^{1 / 2},
$$

where $i$ is the stellar aspect angle. Depending the inclination $i$, $i \simeq 70^{\circ}$ (Vinicius et al. 2006) or $i \simeq 50^{\circ}$ (Domiciano de Souza et al. 2003), we have: $\left(R_{\text {eq }} / R_{\text {pole }}\right)_{\text {phys }} \simeq 1.64$ or 1.46 , respectively, which indicate either near-critical or beyond critical, limiting flattening, established by a rigid, stellar rotation. Similar conclusions are derived if we replace the elliptical shape of the star by a Roche potential. The star is then either a neat, differential rotator, or the interferometric measurements are strongly affected by some circumstellar emission, as proposed by Vinicius et al. (2006). "A neat differential rotator" is a star that has an energy ratio $\tau=K /|W|$ ( $K=$ total kinetic rotational energy; $W=$ graviatational potential energy) larger than expected for a rigid critical rotator, which for a star with Achernar's mass and age at the end of the Main Sequence phase is $0.015 \lesssim \tau_{\mathrm{rc}} \lesssim 0.009$ (Zorec et al. 1988, 2007).

The observed, spectral energy distribution of Achernar is similar to that of a non-rotating star with $T_{\text {eff }}=15000 \pm$ $300 \mathrm{~K}$ and $\log g=3.47 \pm 0.10 \mathrm{dex}$. Since we have no specific proof that Achernar's internal, neat, differential rotation exists, we adopt a conservative approach and assume that the star is a critical, rigid rotator. The apparent, effective temperature, when converted to that of a non-rotating object of similar mass, is then $T_{\text {eff }}=17280 \pm 340 \mathrm{~K}$ or $T_{\text {eff }}=18560 \pm 340 \mathrm{~K}$, for $i=50^{\circ}$ and $i=70^{\circ}$, respectively. For these inclinations, the polar, local, effective temperature is $T_{\text {pole }}=19320 \pm 380 \mathrm{~K}$ or $T_{\text {pole }}=20750 \pm 420 \mathrm{~K}$ (Frémat et al. 2005; Vinicius et al. 2006). Preferring neither one of the two inclinations, we adopted the average value $\left\langle T_{\text {pole }}\right\rangle=20000 \pm 400 \mathrm{~K}$. In a critical, rigid rotator, the equatorial, effective gravity $g_{\mathrm{eq}} \rightarrow 0$, which implies that the local, effective temperature can be $T_{\text {eff }} \lesssim 7500$. For these conditions, the von Zeipel gravity index drops from 1 to $1 / 3$ (Claret 1998 b), so that there is an equatorial region with a fairly uniform effective temperature $T_{\text {eff }} \approx 7500 \mathrm{~K}$, which was adopted in our calculations. The gravity-darkening effect is then calculated using:

$T_{\text {eff }}(\theta)=T_{\text {pole }} g_{\text {eff }}^{\beta / 4}$,

where $\beta=1$ for $T_{\text {eff }}(\theta) \gtrsim 7500 \mathrm{~K}$ and almost $\beta=0.3$ for $T_{\text {eff }}(\theta) \lesssim 7500 \mathrm{~K}$, and $\theta$ is the stellar colatitude, $\beta$ varies with temperature in a continuous way as it is interpolated in the tables of Claret (1998a).

Details of the energy-distribution calculation can be found in Frémat et al. (2005). The spectrum is given primarily by:

$L_{\lambda}(i, \omega)=2 \int_{0}^{\pi / 2} \mathrm{~d} \phi \int_{0}^{\pi} R^{2}(\theta) I_{\lambda}(\mu, \omega) \frac{|\mu|}{\cos \delta} \sin \theta \mathrm{d} \theta$

where $R(\theta)$ is the co-latitude, $\theta$-dependent, distance between the stellar center and the surface of the rotation ally-distorted star; $\mu=\mu(\phi, \omega)=\hat{n} \cdot \hat{\beta}$, where $\hat{n}$ is the unit vector normal to the stellar surface, and $\hat{B}$ is the unit vector that represents the direction of the line of sight; $\cos \delta(\theta, \omega)=-\hat{n} \cdot \hat{r}[\hat{r}$ is the unit vector in the direction of $R(\theta)$ ]; $I_{\lambda}(\mu, \omega)$ is the $\mu$-dependent, monochromatic, specific radiation intensity calculated for the local effective temperatures $T_{\text {eff }}(\theta, \omega)$ and surface gravities $g(\theta, \omega)$. The integration of Eq. (8) was performed using a Gauss-biquadrature of degree 96 in both angles $\theta$ and $\phi$, which is equivalent to a grid of 36481 surface elements of local, plane-parallel, model atmospheres.

To evaluate each local specific intensity $I_{\lambda}(\mu, \omega)$, models were computed in two steps. The temperature structure of the atmospheres was derived as described by Castelli \& Kurucz (2003), using the ATLAS9 computer code (Kurucz 1993). NonLTE level atomic populations were computed using TLUSTY (Hybeny \& Lanz 1995), keeping the temperature and density distributions, derived using ATLAS9, fixed.

We note that the energy distribution of a rapid rotator is slightly bluer, mainly in the far-UV, compared to an object that does not rotate and has the apparent, fundamental parameters of a rapid rotator. If we compare the two model, flux distributions in the visible, spectral range, we notice in the far-IR that the distribution of the rapid rotator, calculated for the Achernar's mass, exhibits a slight flux-deficiency relative to the non-rotating one, which amounts to $0.06 \mathrm{mag}$ in the $J-K$ region, as shown in Fig. 3 (fluxes are in erg $/ \mathrm{cm}^{2} \mathrm{~s} \AA$ and $\lambda$ in $\AA$ ). The difference in the $V-K$ color index is small $\Delta(V-K)=(V-K)_{\Omega=0}-(V-K)_{\Omega=0.9}=$ $0.024 \mathrm{mag}$. This implies that a small, flux excess, originating in a tiny circumstellar envelope, can fill-up the actual flux distribution of the fast rotator and mask an apparent normal, non-polluted, energy distribution.

To simulate the stellar surface of Achernar and the circumstellar environment of the closest Be stars, we modified the original SIMECA code. Since this star is the closest Be star (44 pc) and its circumstellar environment might not be significantly extended $\left(\$ 30 R_{*}\right)$, we refined the grid around the central star and decreased the maximum possible extension of the envelope to $28 R_{*}$, which can reach up to $100 R_{*}$ in the original version.

\section{Disk and/or wind scenario?}

Kervella \& Domiciano analyzed the complete visibility data set of Achernar and demonstrated that the distribution of the measured visibility, around the pole, did not follow a uniform disk. 

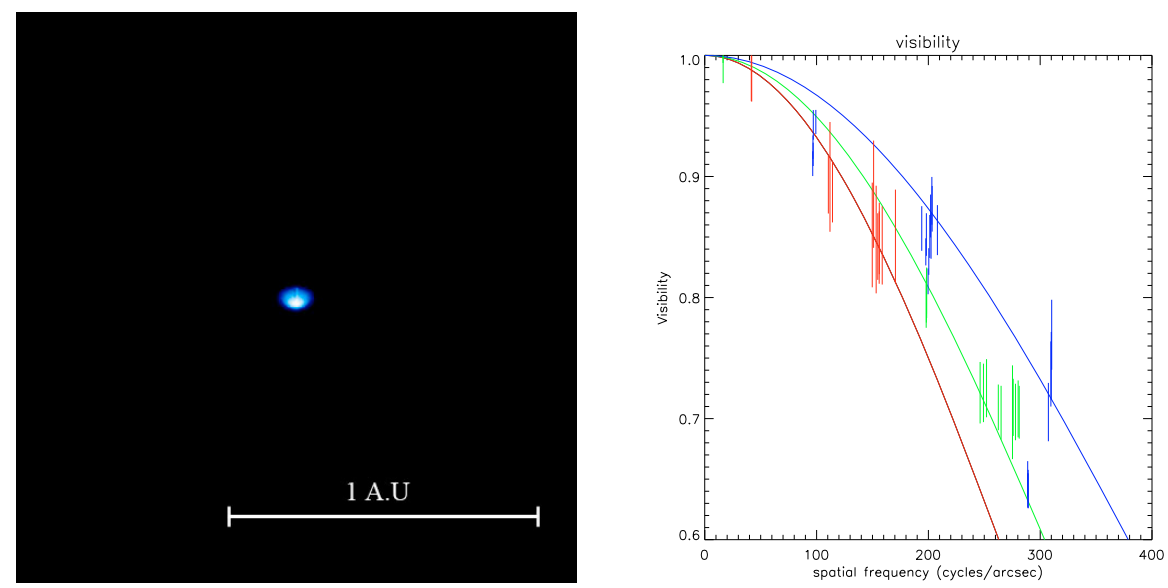

Fig. 1. Left: intensity maps in the $K$-band computed using the SIMECA code, for Achernar's photosphere only (inclination angle of $50^{\circ}$ ). We note that the south pole appears brighter due to its rapid rotation, right: its visibility plotted around the pole (blue), $45^{\circ}$ (green), equator (red), using the VLTI/VINCI data: blue dots (pole $\pm 5^{\circ}$ ), green dots $\left(45^{\circ} \pm 5^{\circ}\right)$, red dots (equator $\pm 5^{\circ}$ ).

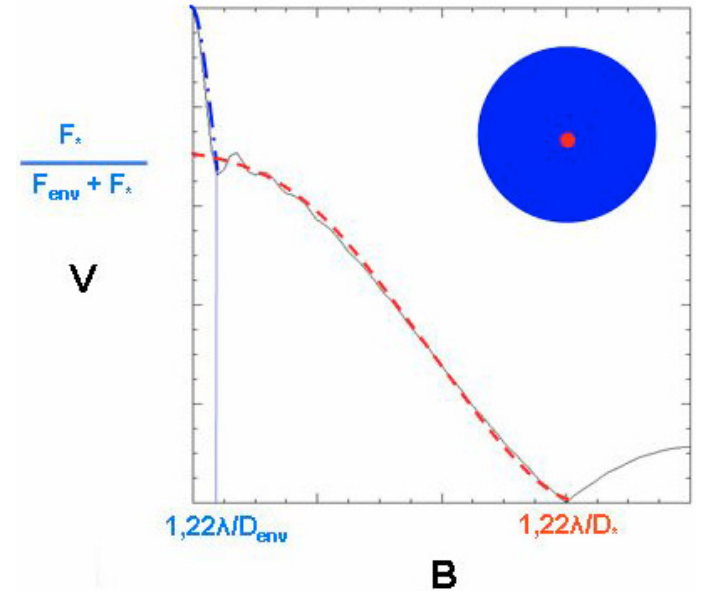

Fig. 2. Schematic diagram of a simple star+uniform envelope model with the corresponding visibility (thin line) as a function of the baseline. The envelope contribution is given by the blue dash-dotted line whereas the star contribution is given by the red, dashed line. We note the "switch off" or "plateau" at the flux ratio $F_{\star} /\left(F_{\text {env }}+F_{\star}\right)$.

There is a clear "switch-off" of visibility on small baselines that KD interpreted in terms of a polar wind.

To attempt to understand this "switch-off", we introduced a simple model (see Fig. 2 with a star (flux: $F_{\star}$, diameter: $\left.D_{\star}\right)+$ a uniform envelope (flux: $F_{\text {env }}$, diameter: $\left.D_{\text {env }}\right)$ ). This simple scheme corresponds approximately to the Achernar visibilities measured by KD. As can be seen in Fig. 2, the measured visibility corresponds to two components: a resolved star (red curve) and an over-resolved envelope (blue line). We note that the envelope is resolved on short baselines, given by $1.22 \lambda / D_{\text {env }}$, whereas to resolve the central star we need baselines of the order of $1.22 \lambda / D_{\star}$. Short baselines are therefore required to constrain fully the shape and extension of the circumstellar disk. We can also estimate the flux ratio between the central star and the total flux, given by $F_{\star} /\left(F_{\text {env }}+F_{\star}\right)$, from the same graph. The "switch-off" derived by KD is typical of an extended object that is resolved by the interferometer.

It is not, however, clear if there is an equatorial extension in their data, since there is no clear "switch-off" in the equatorial direction, whereas a clearer signal is seen along the polar axis. They concluded that the VLTI/VINCI data of Achernar

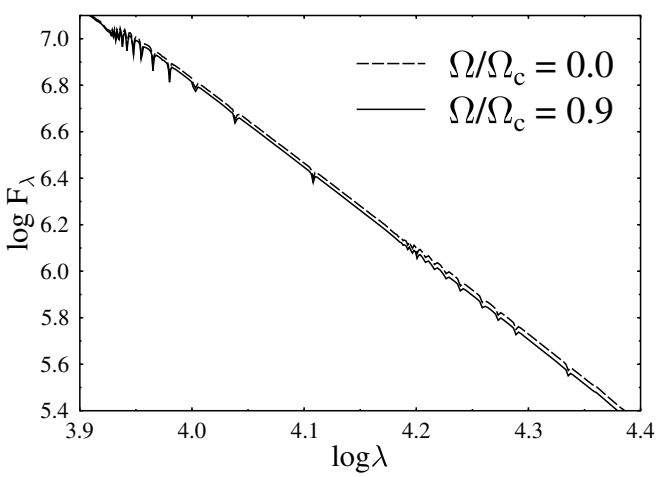

Fig. 3. Slight apparent, flux-deficiency in the far-IR spectral region of a rapid rotator (full line; $\Omega / \Omega_{\mathrm{c}}=0.9$ and $i=70^{\circ}$ ) as compared to that of a star with uniform apparent $\left(T_{\text {eff }}, \log g\right.$ ) parameters (dashed line), when both SEDs are superimposed in the visible spectral range. The normalization of the curves is calculated for the $\lambda_{V}=5556 \AA$ wavelength.

were consistent with the presence of a flattened star with a polar wind.

To investigate these issues concerning the disc and/or wind around Achernar, a complete, astrophysical model that is able to explain simultaneously all spectroscopic and interferometric observations, is required. In the following, we adopt an orientation of the minor axis of Achernar, which is assumed to be the polar axis, of $39 \pm 1^{\circ}$, as suggested by Domiciano de Souza et al. (2003) and in agreement with polarimetric measurements of $37 \pm 8^{\circ}$ by Mc David (2005), although we note that the minor axis is aligned in a vertical direction in Figs. 1, 4, 6, and 8.

To test the scenario proposed by KD, we computed the visibilities and corresponding $\chi_{v}{ }^{2}$ for three different kinds of models, using the SIMECA code:

1) A disk-like envelope model: in this scenario, the most appropriate synthetic visibilities computed by SIMECA for two models of different disk opening angles (different $m 1$ ), are plotted in Fig. 4. We tested more than one hundred different models with different opening angles, densities, and various disk parameters without finding complete agreement with the observed VLTI/VINCI data. In particular, it can be seen, in the lower row in Fig. 4, that our synthetic equatorial visibilities, at short baselines (red vertical line segments), imply a smaller equatorial extension $(m 1<10)$. Thus, we 

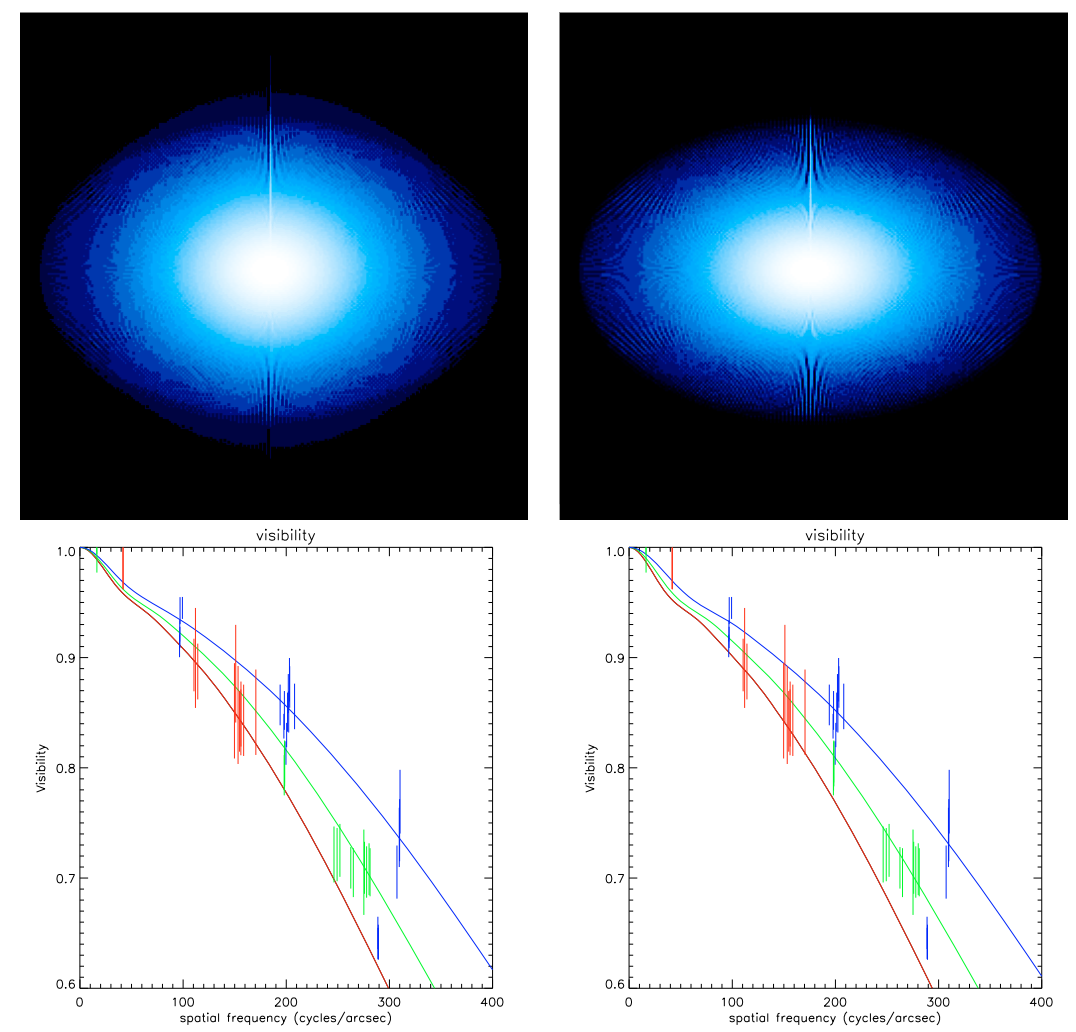

Fig. 4. Upper row: intensity maps in the $K$-band computed using the SIMECA code with parameters from Table 1 showing two models of an equatorial disk with $m 1=10$ (left) and $m 1=1000$ (right). Lower row: their respective visibilities fitted around the pole blue), $45^{\circ}$ (green) and the equator (red) using the VLTI/VINCI data: blue dots (pole $\pm 5^{\circ}$ ), green dots $\left(45^{\circ} \pm 5^{\circ}\right.$ ), red dots (equator $\pm 5^{\circ}$ ).

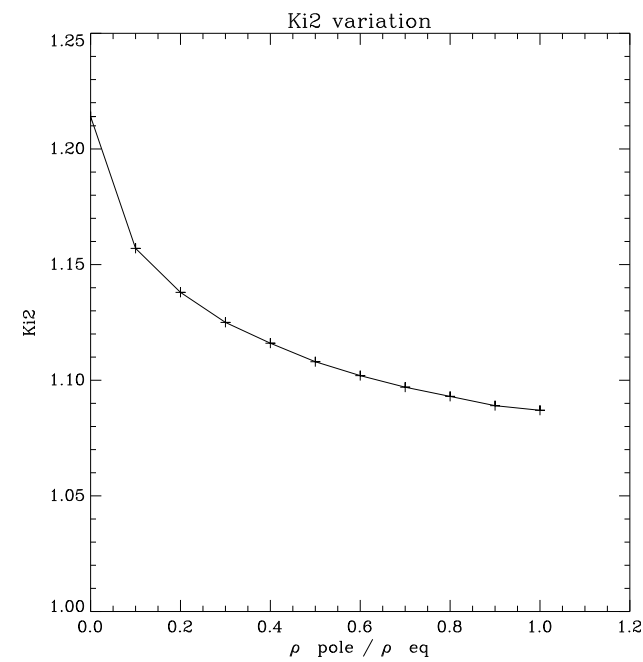

Fig. 5. Reduced $\chi_{v}{ }^{2}$ values obtained for a disk model decreasing when going from a thin, equatorial disk to a more spherical, circumstellar envelope using different $m 1$ values.

were forced to exclude this first scenario for the epoch of the VINCI observations. We obtained $\chi_{v}{ }^{2} \geq 1$ for all our disk-like models as seen in Fig. 5. Since the VLTI/VINCI data provides clear support of a polar-wind contribution, the $\chi_{v}{ }^{2}$ values decrease as the wind asymmetry varies from a purely-equatorial, thin disk to a more spherical, circumstellar geometry, which mimics a stellar-wind contribution along the polar axis. The values of $\chi_{v}{ }^{2}$ measured globally are larger for these models than the pure-wind model presented next.
2) A polar wind model: the visibilities computed using SIMECA for several wind models of various opening angles are in good agreement with the observed VLTI/VINCI data as can be seen in Fig. 6 (lower row). The $\chi_{v}{ }^{2}$ values obtained for our best-fit models are less than 1.0, with a minimum value of 0.88 , for an opening angle around $20^{\circ}$ (see Fig. 7). Thus, a distorted central star with a polar wind appears to be a possible scenario for Achernar, as proposed by KD.

3) A disk+wind scenario: the visibilities computed using SIMECA for our best-fit models are also in agreement with the VLTI/VINCI data, as can be seen in Fig. 8 (right graph) and the corresponding $\chi_{v}{ }^{2}$ in Fig. 9. As expected, $\chi_{v}{ }^{2}$ decreases for a decreasing equatorial disk size, but remains higher than the minimum value measured for the wind model only (0.88). We were unable to obtain solutions for disk sizes smaller than five stellar radii due to numerical instabilities in the SIMECA code. Extrapolating the curve in Fig. 9 when the disk size goes to zero, we measure a $\chi_{v}{ }^{2} \simeq 0.9$, which is higher again than those obtained for the wind scenario. We note that for this scenario the wind, opening-angle, along the poles in Fig. 8, is still about $20^{\circ}$, in agreement with our finding for our second model scenario.

For the 3 scenarios presented, we fix the flux ratio between the disk, wind, disk+wind, and the central star to be approximately $5 \%$. We find that the disk model is less appropriate than the wind model. It is, however, more difficult to distinguish the polar wind and disk+wind scenarios, when the disk is smaller than $10 R_{\star}$ for the disk+wind model. This result agrees completely with that of Carciofi et al. (2008), who showed that the strong, photospheric flattening revealed by the VINCI visibilities on long baselines, could be explained by a critically-rotating star, surrounded by a small disk with $R<2 R_{\star}$. We note that these authors achieved a 

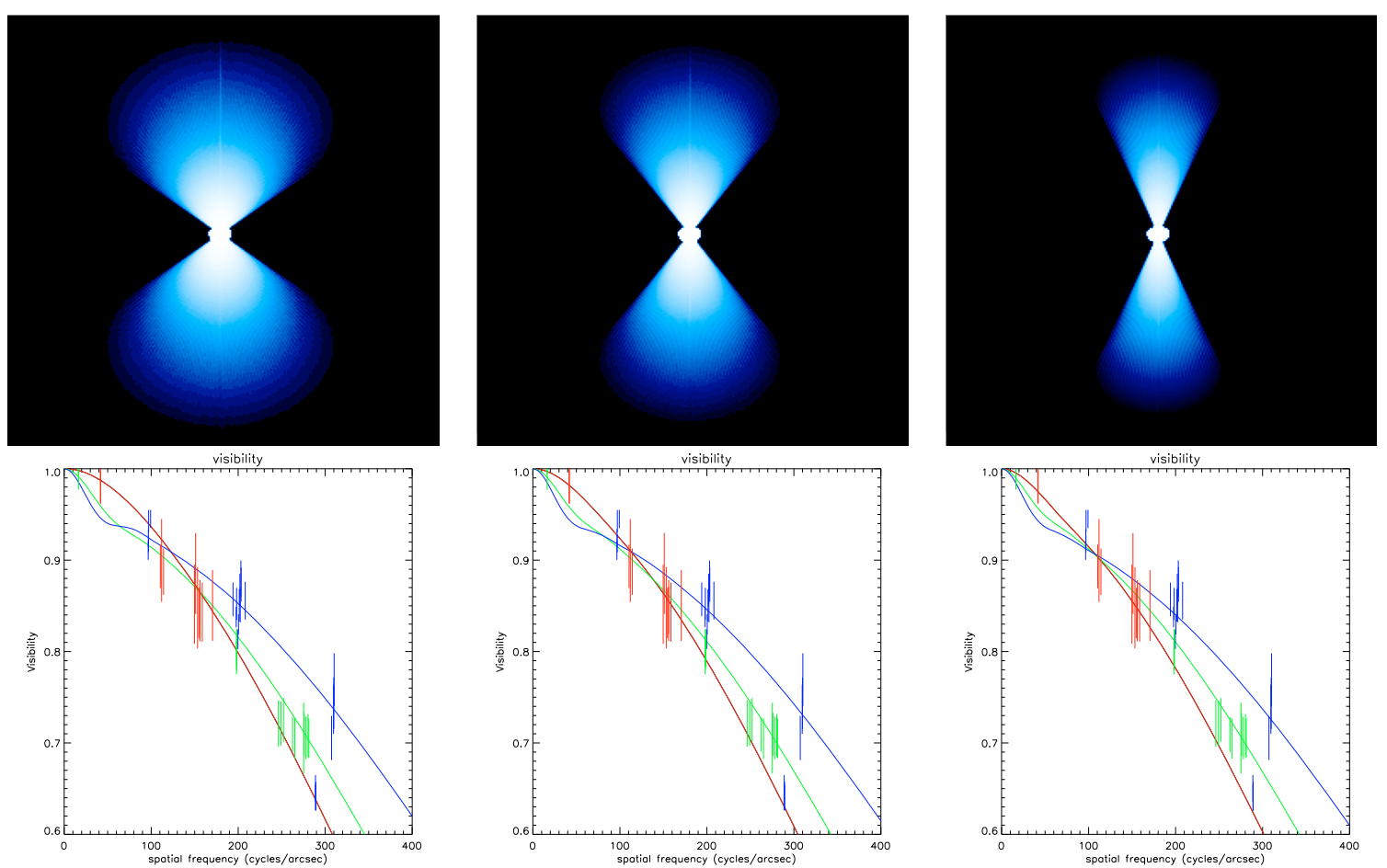

Fig. 6. Upper row: intensity maps in the $K$-band computed using the SIMECA code, for three wind models of different opening angles (respectively from left to right $80^{\circ}, 60^{\circ}, 40^{\circ}$ ). Lower row: their respective visibilities plotted around the pole (blue), $45^{\circ}$ (green), equator (red), using the VLTI/VINCI data: blue dots (pole $\pm 5^{\circ}$ ), green dots $\left(45^{\circ} \pm 5^{\circ}\right.$ ), red dots (equator $\pm 5^{\circ}$ ).

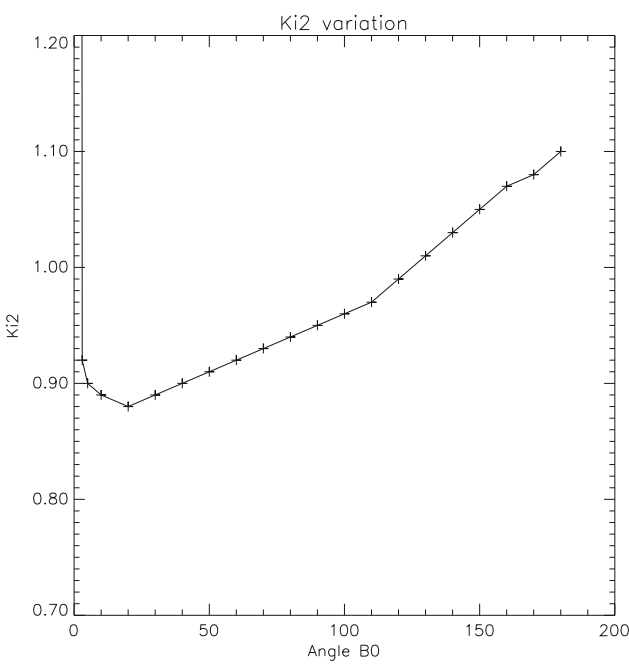

Fig. 7. Reduced $\chi_{v}{ }^{2}$ values obtained for a wind model without any disk contribution, for various wind opening angles, showing a minimum value for an opening angle around $20^{\circ}$.

good fit for visibilities without the need for a polar wind. We find, however, that Achernar's polar extension must be larger than $10 R_{\star}$ to be able to fit the interferometric data satisfactorily and that the minimum $\chi_{v}{ }^{2}$ obtained was only for the wind scenario. We note that there is almost no IR excess in the SED, which is dominated by the central star emission, and that the total IR-excess flux is similarly small for all the models presented in this paper. At the time of the VINCI observations, Achernar therfore appears to have no equatorial disk but instead a confined, polar, stellar wind with an opening angle of approximately 20 degrees. We admit that due to the lack of interferometric measurements on small baselines, this wind, opening-angle must be taken cautiously: it originates in our modeling but is not strongly constrained by the VINCI data.

\section{Evidence for an equatorial disk formation and dissipation}

Since we cannot determined only from the VLTI/VINCI interferometric measurements whether the circumstellar environment of Achernar consist of a pure polar wind or a polar wind with an equatorial disk, we have searched in the litterature other evidences for the presence or not of a circumstellar equatorial disk in the past since, as already outlined in the introduction, Be stars are strongly variable and the disk may forms or dissipates as a function of time.

\subsection{Spectroscopic data}

Vinicius et al. (2006) found evidence of a circumstellar envelope around Achernar based upon the $\mathrm{H} \alpha$ line-profile observed from 1991 to 2002. Since the 1999 line-profile (Fig. 10) exhibits a maximum absorption, they assumed that it was a "true" stellar, photospheric, absorption profile and subtracted it from all remaining $\mathrm{H} \alpha$ lines observed. We note that the $\mathrm{H} \alpha$ profile in Fig. 10 shows inflection points (slope changes) close to the radial velocities -280 and $+300 \mathrm{~km} \mathrm{~s}^{-1}$, which suggest that residual emission could still be present in this spectrum. The $\mathrm{H} \alpha$-line components obtained (Fig. 11) reveal residual emission. This residual emission was interpreted by Vinicius et al. (2006) as an equatorial disk and they discussed the dissipation of the disk between 1993 and 2000 and its formation. Achernar's SED does not appear to exhibit any IR excess (see Fig. 12) compared to 

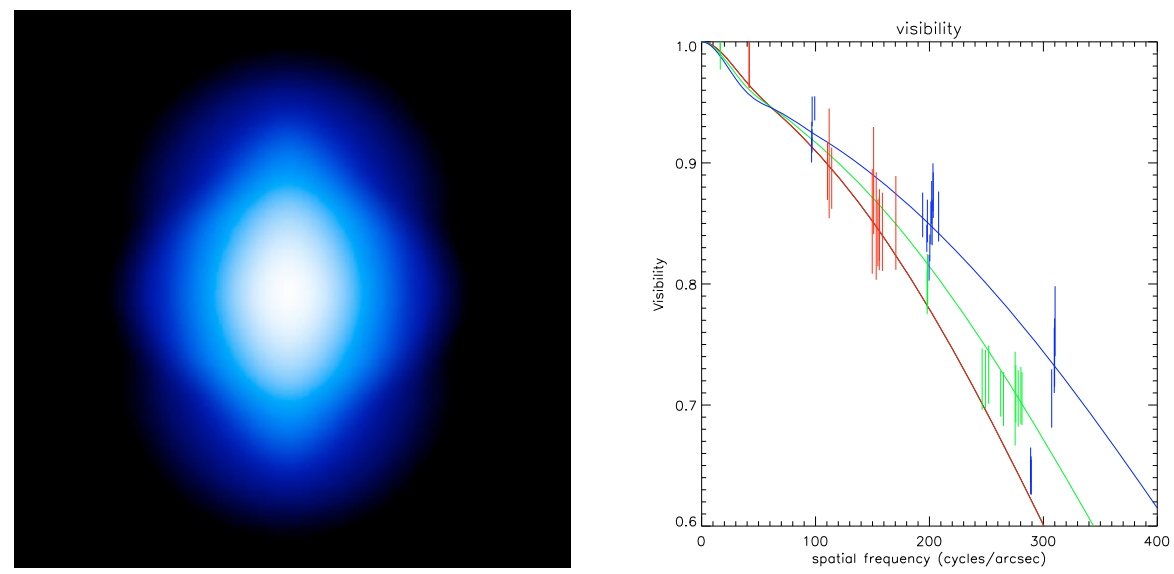

Fig. 8. Left: intensity maps in the $K$-band computed using the SIMECA code showing the best model of a disk+wind envelope, right: its visibility plotted around the pole (blue), $45^{\circ}$ (green), equator (red), using the VLTI/VINCI data: blue dots (pole $\pm 5^{\circ}$ ), green dots $\left(45^{\circ} \pm 5^{\circ}\right.$ ), red dots (equator $\pm 5^{\circ}$ ).

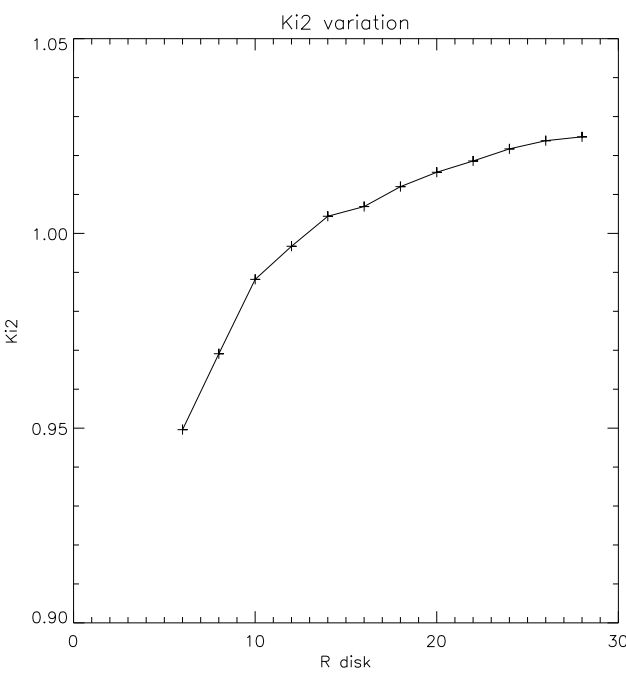

Fig. 9. Reduced $\chi_{v}{ }^{2}$ values obtained for a wind+disk model as a function of the equatorial-disk, outer boundary in stellar radius for an almost constant polar-wind, opening-angle (of about $20^{\circ}$ ).

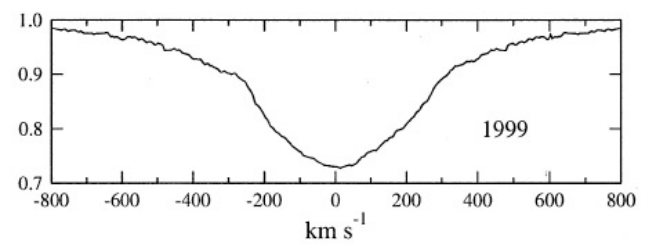

Fig. 10. Achernar $\mathrm{H} \alpha 1999$ line-profile from Vinicius et al. (2006). Since it corresponds to the maximum of absorption for the 1991-2002 period, this line-profile was considered to be the stellar photospheric absorption line.

a classical Be star, which can place strong constraints on the $\mathrm{CE}$ density distribution, i.e. it must remain very small.

As already mentioned, the $\mathrm{H} \alpha$ line was almost always in absorption, but also showed strong morphological and equivalentwidth $(\mathrm{EW})$ variations. These variations can be related to a modification of a weak, circumstellar-envelope, emission. They determined that there was almost no emission in 1999, and that the $\mathrm{H} \alpha$ line profile observed at this time corresponded to the stellar, photospheric, absorption-line (Fig. 10). By subtracting this

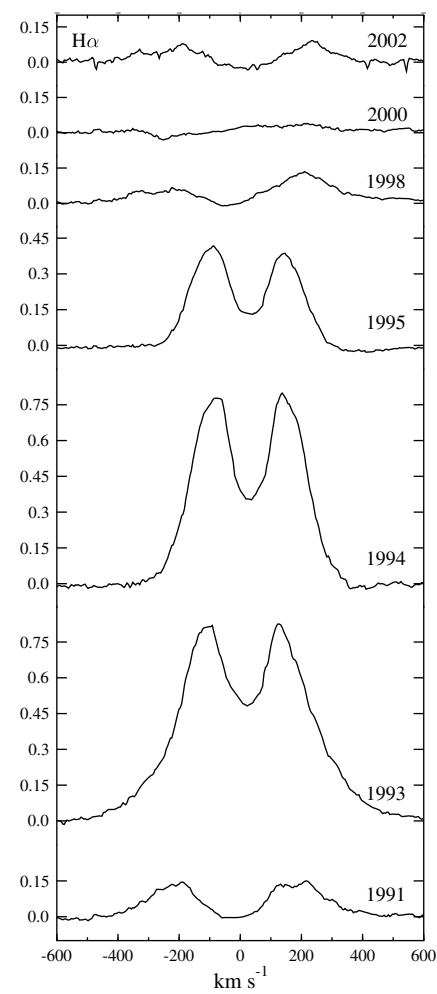

Fig. 11. H $\alpha$, "emission-only", line-profiles from 1991 to 2002 obtained after subtraction of the 1999 (Fig. 10) photospheric absorption profile.

1999 profile from other line profiles, they obtained the residual, emission profiles for all measurements between 1991 and 2002 .

All of the emission-line profiles are strongly double-peaked (apart from in 1998 and 2000) and the double-peak separation (DPS) varies between 210 and $450 \mathrm{~km} \mathrm{~s}^{-1}$. This double-peaked structure in emission lines is usually associated with a rotating disk, although such a profile can also be produced by velocities associated with a polar wind (Stee \& Araújo 1994). Nevertheless, the velocities measured with the double-peak separation are inconsistent with a polar wind model since terminal velocities of classical Be star are very high, about $1000 \mathrm{~km} \mathrm{~s}^{-1}$. Taking an inclination angle of $50^{\circ}$, this would have produced a DPS of around $1300 \mathrm{~km} \mathrm{~s}^{-1}$, which is far above the upper limit observed. Moreover, considering that Achernar's projected 


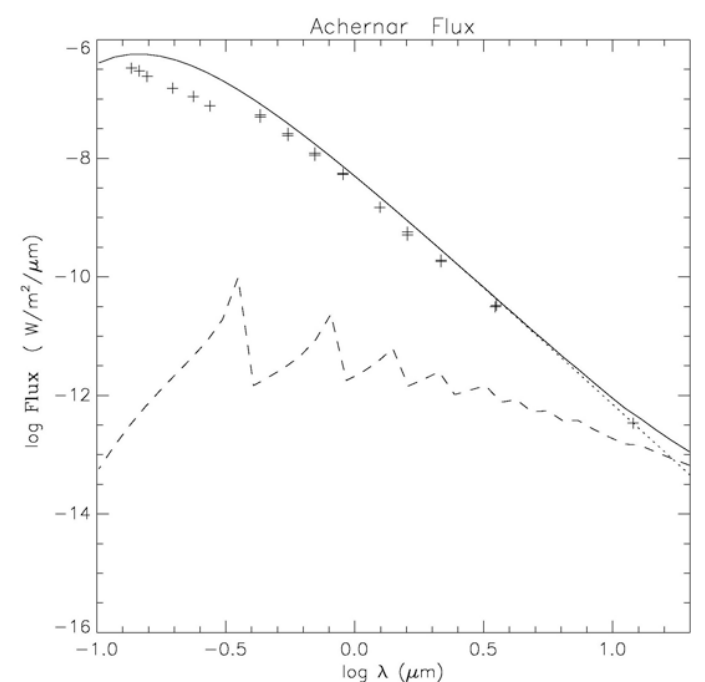

Fig. 12. Achernar Spectral Energy Distribution (SED). Dotted line: stellar contribution. Dashed line: envelope contribution. Solid line: total flux. Crosses: data points from the SIMBAD database. Note that for all models discussed in the paper we checked that the SED stays always in agreement with the data points.

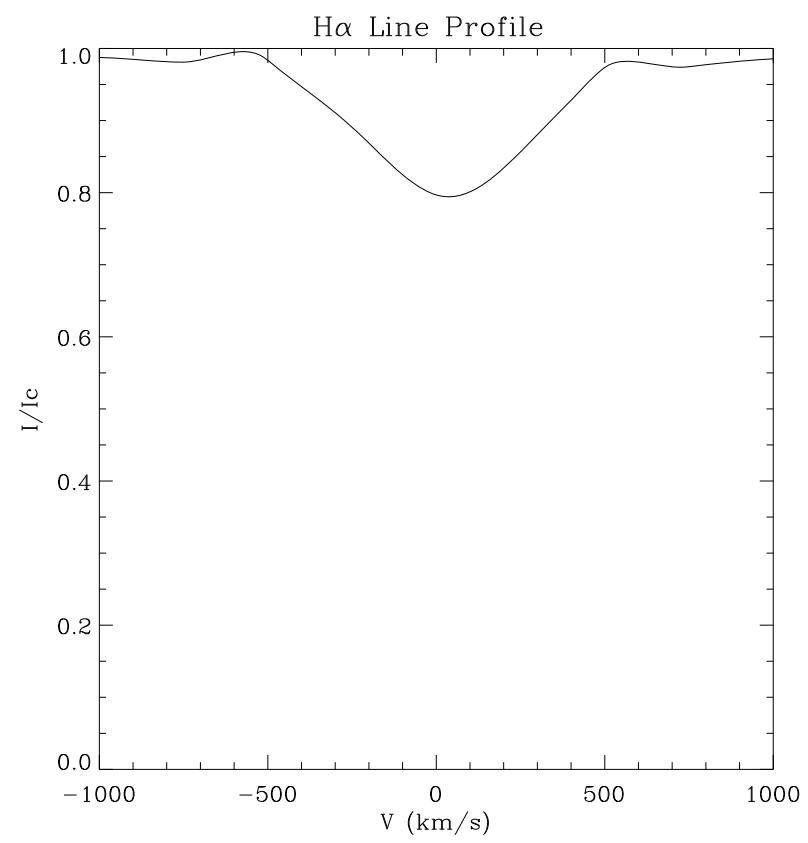

Fig. 13. Typical $\mathrm{H} \alpha$ line-profile computed using SIMECA with the basic parameters given in Table 1 for the various scenarios described in Sect. 4.

rotational velocity $V \sin i$ is $230 \mathrm{~km} \mathrm{~s}^{-1}$ and that the putative circumstellar equatorial disk is smaller than $10 R_{\star}$, the corresponding DPS for a rotating, equatorial disk would be between $144 \mathrm{~km} \mathrm{~s}^{-1}$ (for a $10 R_{\star}$ uniform disk) and $460 \mathrm{~km} \mathrm{~s}^{-1}$ (i.e. $2 V \sin i$ ), which is fully compatible with the measured values.

Additional evidence for the presence of a small, circumstellar disk is the correlation between the line-profile intensity and the DPS presented in Fig. 14. We note that there is already a known power-law relation between $\mathrm{H} \alpha$ equivalent width (corrected for the absorption component) and DPS for Be stars (Zamanov et al. 2001). In general it is also better to use $\mathrm{H} \alpha$ equivalent-width measurements instead of maximum intensity, because the latter depends on spectral resolution and is more

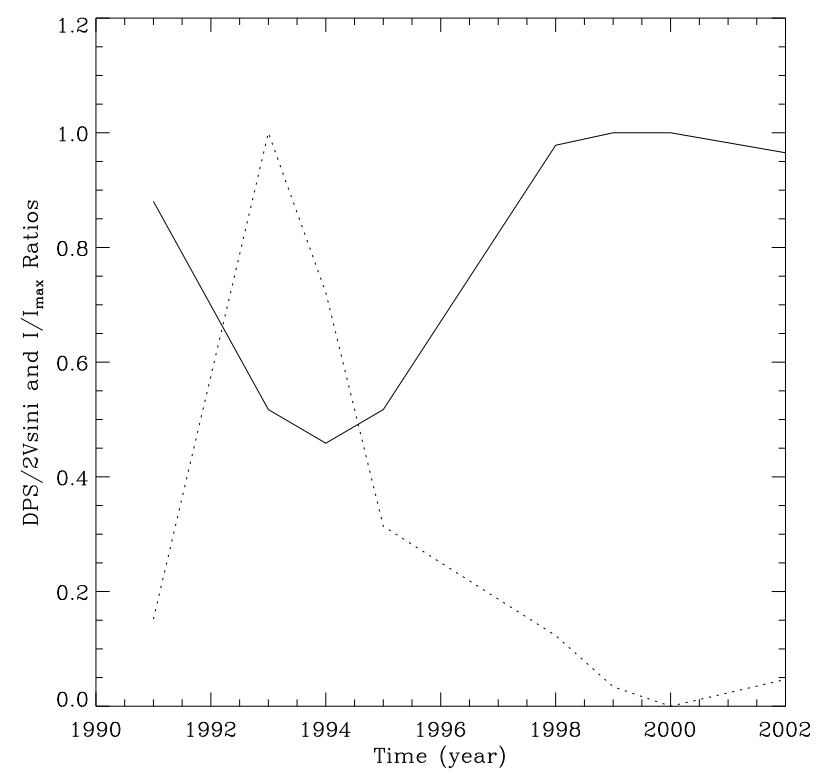

Fig. 14. Solid line: $\mathrm{H} \alpha$ Double-Peak Separation (DPS), normalized by $2 V \sin i$, and plotted as a function of time for the 1991-2002 Vinicius et al. data period. Dotted line: $\mathrm{H} \alpha$ line-profile intensity normalized by the maximum intensity residual from Fig. 11 (in 1993), and plotted as a function of time for the same period.

sensitive to structure within the profile. Since our model uses an axisymmetric geometry, line asymmetries are not, however, be reproduced and we choose to follow the line-profile intensity variation. For profiles with unequal-intensity, blue and read peaks, we choose to assume that the maximum of the two peaks is the line intensity.

When the emission is weak (i.e. 1991, 1998, 2002), the DPS is close to twice the value of the stellar $V \sin i$, whereas when the emission is stronger (i.e. 1993, 1994, 1995), the DPS is smaller. We note that $\mathrm{H} \alpha$ emission was re-emerging in 2002, around the time of the VINCI/VLTI observations. This can be interpreted as variations in the disk, global extension. Since the $\mathrm{H} \alpha$ line-profile was observed throughout an entire cycle of variations, we propose the following scenario for the formation and the dissipation of the disk.

In Fig. 15, we plot the $\mathrm{H} \alpha$ intensity, normalized by the maximum of emission (in 1993), as a function of the DPS, normalized by $2 V \sin i$. We can separate the disk creation/dissipation cycle into 3 phases:

- Phase 1: between 1991 and 1993, the DPS decreases as the intensity increases.

- Phase 2: between 1993 and 1995, the intensity decreases as the DPS remains nearly constant.

- Phase 3: between 1995 and 1999, the DPS increases again as the intensity slowly decreases.

- Phase 1 (again): between 2000 and 2002. The disk is reforming, but in 2002, the $\mathrm{H} \alpha$ line-profile is smaller than in 1991. Assuming that the variation is periodic or pseudo-periodic, we can therefore estimate roughly a pseudo-cycle of about 13 years, in agreement with the average, emission-cycle duration of 11 years measured by Vinicius et al. (2006).

We can estimate the disk radius by assuming that the disk is Keplerian. In this case, its outer radius is given by:

$R_{\mathrm{disk}} / R_{\star}=\left(\frac{2 V \sin i}{\mathrm{DPS}}\right)^{2}$ 


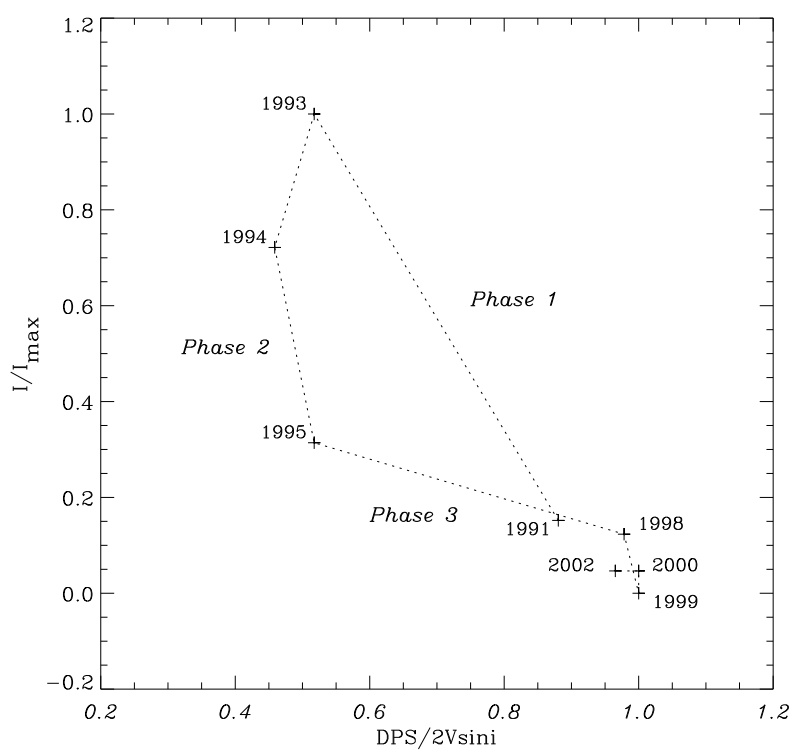

Fig. 15. Normalized intensity as a function of the normalized DPS for the 1991-2002 Vinicius et al. (2006) data period.

Since at the maximum of emission (in 1993), the DPS was $211 \mathrm{~km} \mathrm{~s}^{-1}$, the corresponding disk extension was $4.8 R_{\star}$.

We gathered data between 1960 and 1990, from the literature works of Jaschek et al. (1964), Andrews et al. (1966), Dachs et al. (1977, 1981, 1986, 1992) and Hanuschik et al. (1988, 1996). Since the data quality varies significantly from one reference to another, we were unable to determine the DPS for all of the 1960-1990 data, but only for data since 1973. Since the global $\mathrm{H} \alpha$ line intensities were difficult to determine, we decided to classify the profiles into three categories: lines in absorption; central absorption and weak emission in the wings; and strong emission. Presented in Fig. 16 including data from Vinicius et al. (2006), these data indicate that both the DPS and line-intensity variation appear to be quasi-periodic, with a pseudo-period of between 12 and 16 years. As found by Vinicius et al., the variations in DPS and emission strength appear to be anticorrelated, and the DPS minimum value, in 1979 , of $250 \mathrm{~km} \mathrm{~s}^{-1}$, is close to the 1994 value of $211 \mathrm{~km} \mathrm{~s}^{-1}$.

We note that for all models presented in Sect. 4, we verified that the $\mathrm{H} \alpha$ line-profile, determined using SIMECA, in particular for the polar-wind scenario, was weak and more or less consistent with the observations. A typical, line-profile, with the global parameters from Table 1, is plotted in Fig. 13.

\subsection{D kinematical model}

To test several hypotheses for the disk formation/dissipation processes, we developed a simple, 2D-axisymmetric, kinematical, model, assuming an optically-thin disk and without considering the effects of either disk projection on to the photosphere or disk occultation by the star.

We start by establishing the 1D expansion velocity field, $v_{r}(r)$, in the disk (200 radial points corresponding to $12 R_{\star}$ ). These values can evolve in time but do not depend on any forces, which is the reason that is called kinematic and not a dynamic model. The $1 \mathrm{D}$ density distribution, $\rho(r)$, is calculated for all radii using the continuity equation and assuming $\rho(1)=\rho_{\text {phot }}$. This $1 \mathrm{D}$ density distribution is then used to create a

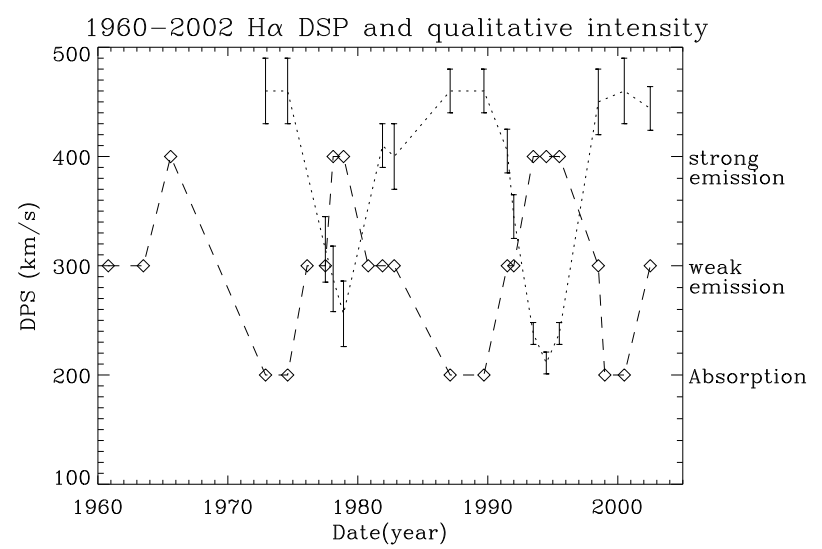

Fig. 16. $\mathrm{H} \alpha$ DPS (dotted line with error bars) and qualitative intensity (dashed line and diamonds) variations between 1960 and 2002 from various authors: Jaschek et al. (1964); Andrews et al. (1966); Dachs et al. (1977, 1981, 1986, 1992); Hanuschik et al. (1988, 1996); and Vinicius et al. (2006).

$2 \mathrm{D}$ axisymetric $(200 \times 200)$ grid. The rotational, velocity-field, $v_{\phi}(r)$ is defined by:

$v_{\phi}(r)=V_{\text {rot }} \cdot\left(\frac{r}{R_{\star}}\right)^{\beta}$.

Where $V_{\text {rot }}$ is the stellar rotational velocity and $\beta$ is the exponent of the rotational-velocity law, $\beta=-0.5$ corresponds to Keplerian rotation, $\beta=-1$ to momentum conservation, $\beta=0$ to constant rotation, and $\beta=1$ to solid body rotation. According to the observations of Meilland et al. (2007), we assumed the Keplerian rotation-law in our modeling.

The projected velocity $V_{x}(r, \phi)$, is then calculated by projecting both $V_{r}$ and $V_{\phi}$ along the line of sight. The emission line-profile is then computed by integrating the emission on an iso-velocity bin between $V_{x}-\delta v / 2$ and $V_{x}+\delta v / 2$, where $\delta v$ depends on the velocity sampling. The reconstructed profile has 200 data points with values of velocity ranging from $-2 V \sin i$ and $2 V \sin i$.

\subsection{Disk formation}

The disk formation depends only on two variables: the mass flux at the equator, and the expansion, velocity-field. Assuming that both are constant and that the expansion-velocity field does not depend on the radius (i.e. $V_{r}(r)=$ const.), we can estimate the expansion-velocity value in the disk. Its creation timescale is about 3-5 years (see Fig. 15), and at the maximum of intensity the disk extension is about $4.8 R_{\star}$, which was obtained in Sect. 5.1 from the DPS, assuming a Keplerian, rotating-disk. The average, expansion-velocity during disk creation is consequently approximately $0.27 \pm 0.08 \mathrm{~km} \mathrm{~s}^{-1}$.

In our modeling, we tested two distinct scenarios for the disk formation. In both scenarios, we consider that the mass flux at the photosphere, $\phi_{M}(0)$, is zero at the beginning of the process. In the first scenario, $\phi_{M}(0)$ goes instantaneously from zero to $\phi_{\max }$ and remains constant throughout the disk formation. In the second scenario, $\phi_{M}(0)$ increases from zero to its maximum value, with an adjustable, power-law and timescale. In both scenarios, we consider that the expansion, velocity-field is uniform and stationary. Its value is fixed to obtain a disk-formation, timescale that is compatible with observations (i.e. 2-3 years). In Fig. 17, 


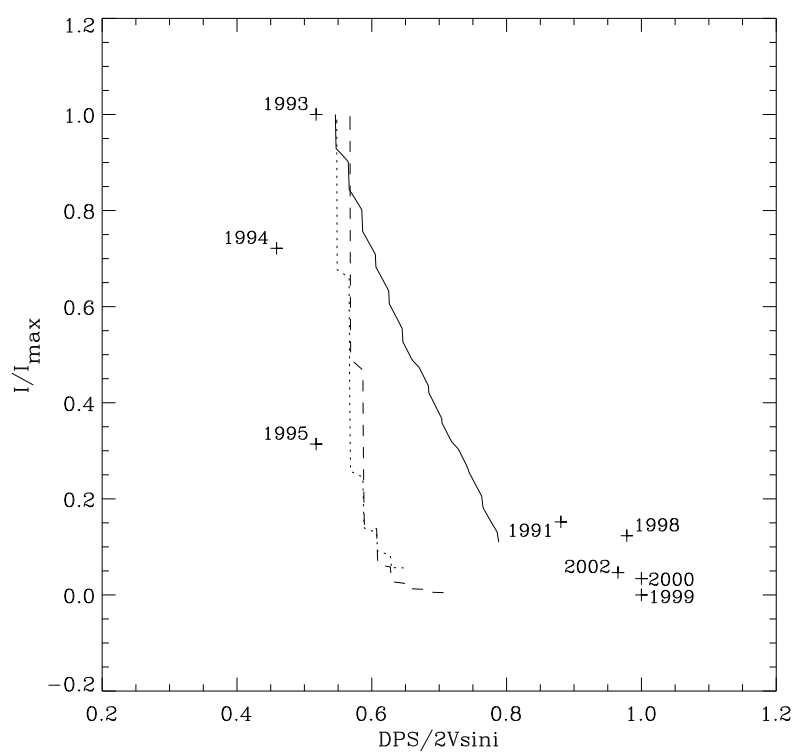

Fig. 17. Normalized DPS versus normalized intensity diagram for various equatorial disk formation processes. Solid line: constant mass-flux. Dotted line: increasing mass-flux as a function of $t^{1}$. Dashed line: increasing mass-flux as a function of $t^{2}$. The + symbols indicates the 1991-2002 data from Vinicius et al. (2006).

we plot the normalized DPS versus normalized-intensity, diagram for these scenarios.

None of these scenarios is able to fit correctly the 1991-1993, disk-formation, observations. The main problem is the inability to reproduce the high DPS observed at the beginning of the disk formation. However, the constant, mass-flux scenario provide a more appropriate fit to the data than the increasing, mass-flux, scenario. A decreasing, mass-flux, scenario may be a possible solution. This point is discussed in more detail in Sect. 5.5. We note, however, that the model and observational comparison of disk growth in Fig. 17, is limited by the lack of data in 1992, so the goodness of the agreement is difficult to assess.

\subsection{Disk vanishing}

We test two classical, disk-dissipation, scenarios. These scenarios and their observational predictions were described by Meilland et al. (2006).

We consider that the disk is stationary, before dissipation begins. The velocity field is assumed to be uniform and stationary. In both scenarios, the mass-flux decreases from its maximum value to zero. The main difference between these scenarii is the mass-flux, decrease, timescale.

In the first scenario, the mass-flux decreases faster than the timescale of propagation through the disk (i.e. $R_{\text {disk }} / v_{r}$ ). Consequently, the under-density zone, which is created by the dramatic decrease in the mass-flux, propagates into the disk and forms a ring-like structure. In Meilland et al. (2006), the decrease was found to be instantaneous, but this is an unnecessary condition to create such structure.

In the second scenario, the propagation through the disk is faster than the timescale of the mass-flux decrease. Thus, we consider the model to be quasi-stationary, i.e. with different $\phi_{M}(t)$ at each step of the simulation. Consequently, the disk becomes fainter and disappears, without any modification to its global morphology and geometry.

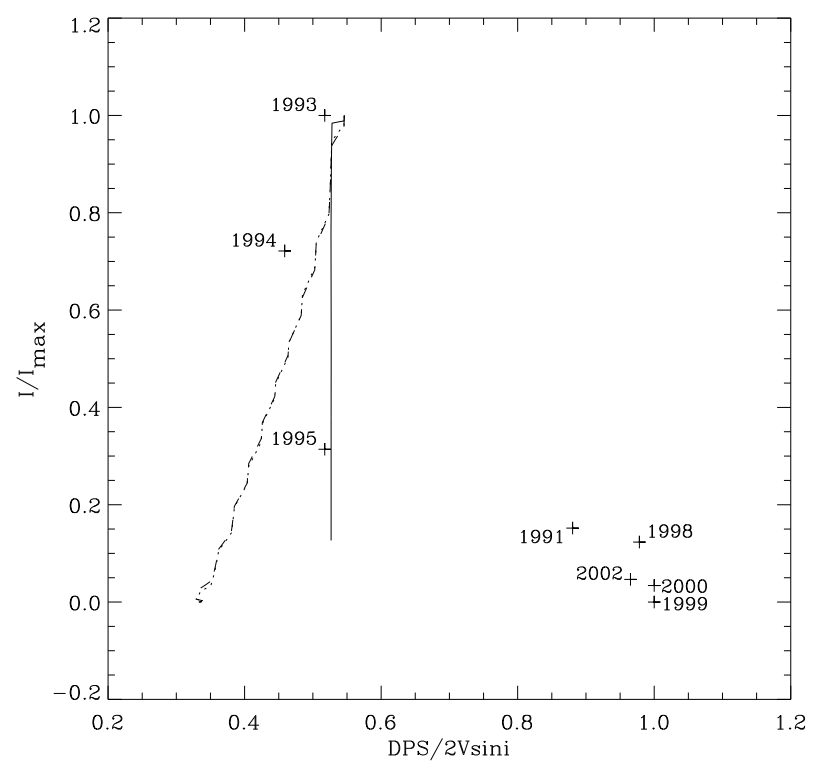

Fig. 18. Normalized DPS versus Normalized intensity diagram for various equatorial disk dissipation scenarios. Solid line: mass-flux decreasing with timescale larger than $V_{r} \times R_{\text {disk }}$. Dotted line: mass-flux decreasing with timescale smaller than $R_{\text {disk }} / V_{r}$. Dashed line: mass-flux intantaneous drop to 0 .

Meilland et al. (2006) measured small differences in the lineprofile modification as a function of time. In the fast-dissipation, scenario, i.e. ring formation, the disk dissipates from the inside, where the rotational velocity is larger. The result is a progressive decrease in the DPS as the line intensity decreases. In the slowdissipation, scenario, however, all of the disk dissipates at similar speed, and the line profile decreases without DPS modifications.

In Fig. 18, we present the normalized DPS versus normalized-intensity diagram, for both scenarios, which do not fully agree with observations. We note that for the second scenario, Fig. 18 illustrates the case of an instantaneous drop to zero mass flux. The polar-density, maps for both scenarios are plotted in Fig. 19.

\subsection{Outburst scenario}

Since the previously-described scenarios were unsuccessful in reproducing the disk-creation, observations, we developed a scenario that explains both phase 1 (formation) and phase 2 (dissipation almost without variation in the DPS) of the line-profile variation. In this "Outburst scenario", $\phi_{M}(0)$ starts with its maximum value and then decreases in value to zero as an adjustable, power-law. The burst propagates in the circumstellar environment, forming a disk structure with a sharp, outer edge (i.e. depending on the mass-flux, decrease, timescale, as in the previous subsection). Such an "Outburst scenario" was proposed to explain the spectroscopic variations of some Be stars (Rivinius et al. 2001).

By adjusting the mass-flux, power-law and the expansion velocity, we reproduce not only the observations of the disk formation (1991-1993) but also the beginning of the disk dissipation (1993-1995). The normalized DPS versus normalized-intensity, diagram is plotted in Fig. 20, using a mass-flux, decrease that follows a $t^{-0.5}$ law, an expansion velocity of $0.2 \mathrm{~km} \mathrm{~s}^{-1}$, and a maximum, disk radius of $6 R_{\star}$. The corresponding density maps for the first 3 years of the burst are plotted in Fig. 21 . 

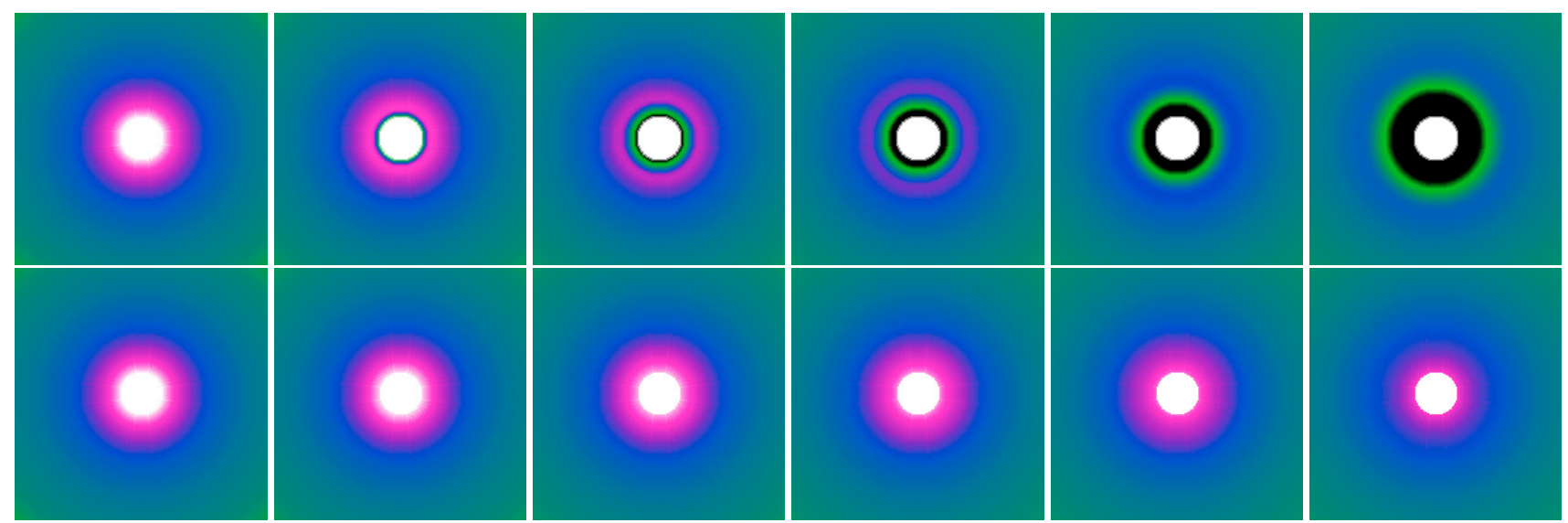

Fig. 19. Density, polar-maps for two different dissipation scenarios. Above: instantaneous mass-flux drop to 0 , creating a ring structure during the dissipation. Below: if the mass-flux drop timescale is sufficiently long $\left(>R_{\text {disk }} / V_{r}\right)$ the dissipation process corresponds to a succession of quasistationary models with a smaller mass-flux. The six pictures correspond to $t=0, t=6$ month, $t=1$ year, $t=1.5$ year, $t=2$ years, and $t=3$ years. The color scale is (in decreasing density order): white, purple, blue, and green. The star is white and the box size is $0.66 \times 0.66 \mathrm{AU}$.

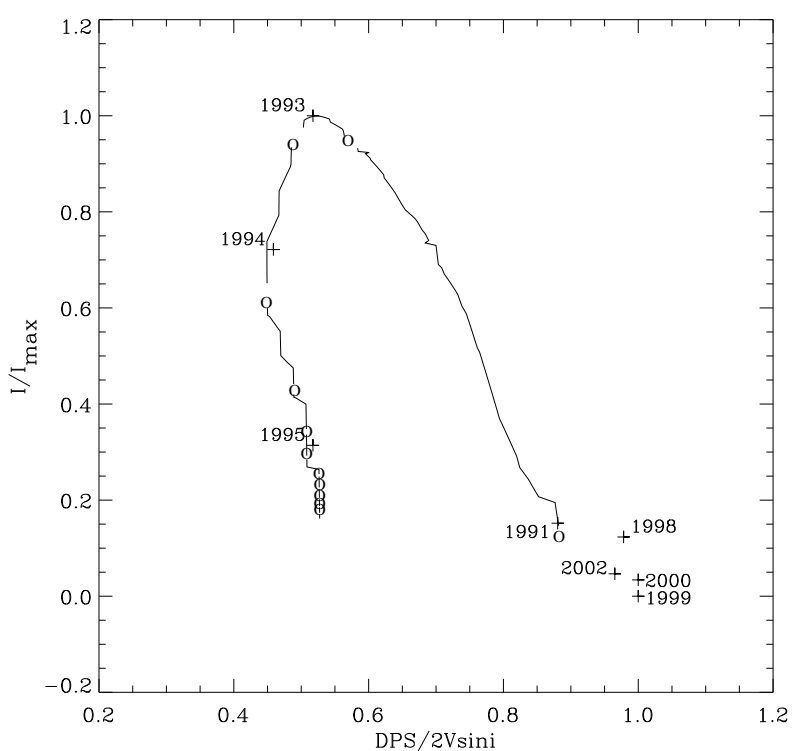

Fig. 20. Normalized DPS versus Normalized Intensity diagram for the Bursting scenario. Each solid line corresponds to one year of the evolution process. The accordance with the measurements is almost perfect for the 1991-1995 period. But this model failed to reproduce the 1995-1999 DPS variation.

The scenario outlined above does not, however, reproduce data for the phase 3 (1995-1999), well. As the intensity continues to drop, the normalized DPS does not increase significantly and converges to a value of 0.6 , whereas the 1998 observation shows a value of about 0.97 . No classical, dissipation scenario was able to reproduce such a variation and an additional physical mechanism must be added to our model before it can be reproduced.

\subsection{Contraction of the disk?}

The phase 3 (between 1995 and 1999) is a major problem in the modelling of Achernar's equatorial, disk formation and dissipation, since the rise in the DPS between 1995 and 1999 cannot be reproduced using a simple, slowly-expanding, model. A possible explanation may be a disk contraction, with a slow decrease in density.
We attempt to reproduce such a model by reverting the velocity field by about 4-5 years, to a time after the burst start in 1994-1995. We tested three methods to reverse the velocity field. In the first approach, which was the most ad-hoc, we reverses the entire velocity field al together. In the second method, we started to reverse the velocity field, from the center of the disk, to the outer edge. Finally in the third method, we started the velocity change at the outer edge and propagate it to the inner edge. The first two scenarios were unable to reproduce the above variation. This is unsurprising, since in these cases, the disk does not really shrink in size, but is depleted out wards from the inside to the outside.

It is very difficult, however, to explain physically such a variation. We assume that circumstellar matter is driven by three forces, the radiative pressure, gravity, and centrifugal force, and that these forces reach equilibrium, before disk dissipation. A small decrease in the radiative force can then induce a negative balance of the forces, and cause the circumstellar matter to re-accrete onto the star. If the decrease occurs first at the photosphere the matter near the star will re-accrete first, but if the decrease in radiative force occurs first at the outer radius of the circumstellar disk, perhaps due to disk opacity, the disk then starts to contract and the matter eventually re-accretes onto the star.

Our results of such a model failed to reproduce the observations, since the contraction of the disk produces an over density that propagated from the outer edge to the center of the disk. The maps of this process were plotted in Fig. 22. The resulting DPS versus intensity diagram, presented in Fig. 23, is not consistent with the observations, since the over-density produces an increase of line-profile intensity, represented by the curly structure in the DPS-versus-Intensity diagram.

We decided to produce a time-dependent, truncation of the disk to fit the observations. This method is entirely ad-hoc; the agreement, however, with the 1995-1999 line-profile, evolution is more appropriate than for the velocity-field reversion, even though not yet ideal. The DPS/Intensity diagram of this scenario is plotted in Fig. 23.

\subsection{Comments on the phase 3 (1995-1999)}

In the phase 3, we must also explain the steady, slight increase in the DPS, as the global, line-emission, intensity, decreases. Two 

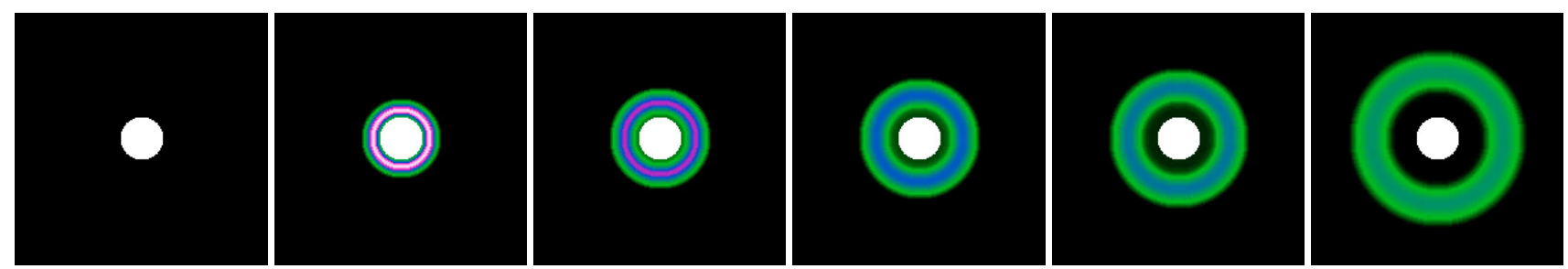

Fig. 21. Density polar maps for the bursting scenario with the same color scale as in Fig. 19. The six pictures correspond respectively to $t=0$, $t=6$ months, $t=1$ year, $t=1.5$ year, $t=2$ years, $t=3$ years. The star is white and the box size is $0.66 \times 0.66 \mathrm{AU}$.
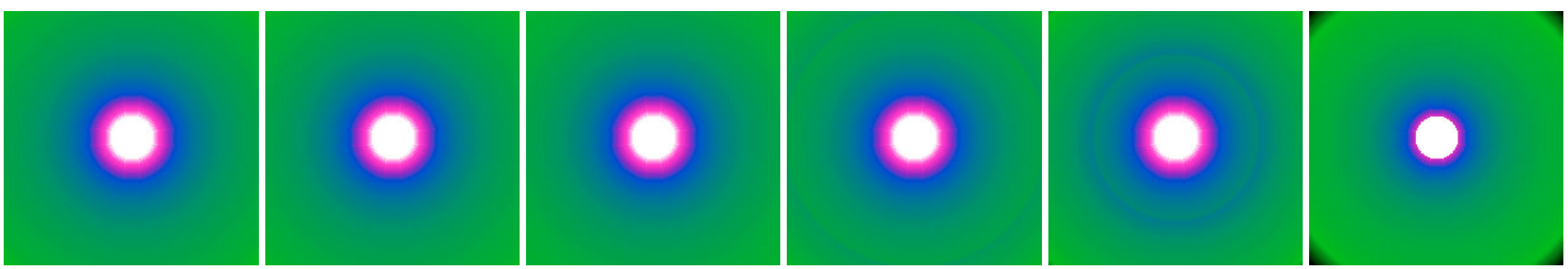

Fig. 22. Density polar maps for the velocity field reversion scenario with the same color scale as in Fig. 19. The six pictures correspond respectively to $t=0, t=6$ months, $t=1$ year, $t=1.5$ year, $t=2$ years, $t=3$ years. An over density wave is propagating from the disk outer edge to the center (in blue). The star is white and the box size is $0.66 \times 0.66 \mathrm{AU}$.

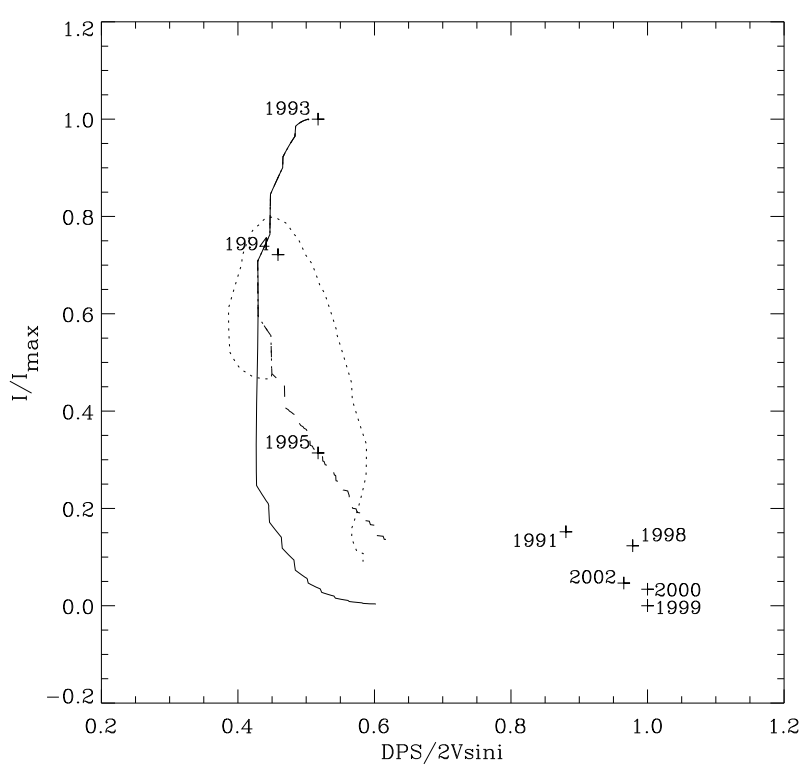

Fig. 23. Normalized DPS versus normalized-intensity diagram for various contraction scenarios. Solid line: outburst scenario with instantaneous reversion of the entire velocity field at $t=4$ years. Dotted line: outburst scenario with progressive reversion of the entire velocity field starting at $t=4$ years. Dashed line: outburst scenario with ad-hoc, disktruncation, starting at $t=4$ years following a linear time dependency.

scenarios could explain this behavior. In one of these scenarios, it is assumed that there is a static, but fairly turbulent ring, whose optical depth varies with time, due to the accumulation of matter ejected by the central star. In the other scenario, the ring rotates around the star, but its radial, optical depth decreases with time, possibly due to a steady widening of the ring diameter caused by the turbulent and thermal pressures in the ring.

The effects on spectral lines produced in the first scenario was studied by Cidale \& Ringuelet (1989) and Chauville et al. (2001). In this case, the emission at each wavelength of the observed line originates in the entire ring, so that the maximum intensity in the emission peaks depends only on the single parameter $\Gamma=\left(S_{\mathrm{H} \alpha} / F_{*}\right) R_{\text {ring }}^{2}$, where $S_{\mathrm{H} \alpha}$ is the line, source-function and
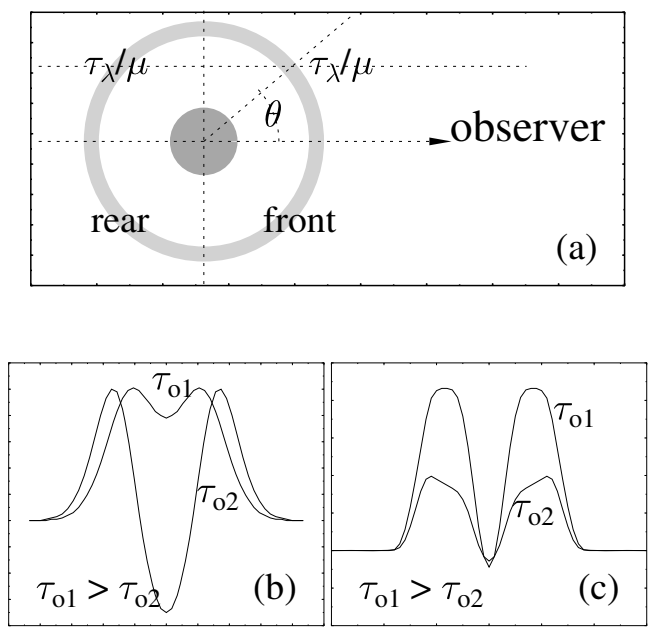

Fig. 24. a) Schematic representation of the star+ring system, where $\mu=$ $\cos \theta$ and $\tau_{\lambda}=\tau_{0} \Phi(\Delta \lambda)$ is the radial ring opacity at the wavelength displacement $\Delta \lambda$; b) line-emission profiles produced by a static ring and for two different ring radial opacities and the same value of $\Gamma$; $\mathbf{c}$ ) lineemission profiles produced by a rotating ring and for two different ring radial opacities and constant $\Gamma$.

$R_{\text {ring }}$ is the radius of the ring. The displacement of a peak from the line center, $\Delta \lambda_{\text {peak }}=\lambda_{\text {peak }}-\lambda_{\mathrm{o}}$, is then controlled by the radial, optical depth of the ring, $\tau_{\mathrm{o}}$. Thus, the $\Delta \lambda_{\text {peak }}$ is measured by assuming that a maximum emission corresponds to the peak, and $\Phi\left(\Delta \lambda_{\text {peak }}\right)=[\ln (2 \Gamma)] / \tau_{\mathrm{o}}$, where $\Phi$ is the line absorption profile. The intrinsic Doppler line-width, of significant size, required to model the line profile, can be reproduced by turbulence in the ring, if we assume that $\Phi$ is Gaussian. The smaller the value of $\tau_{\mathrm{o}}$, the smaller then is the global emission and the larger is the DPS, as depicted in Fig. 24b.

The second scenario was investigated by Arias et al. (2007). In this case, the emission at each wavelength in the observed line comes mainly from the sectors of the ring that have the appropriate radial velocity, i.e. each $\Delta \lambda= \pm \lambda\left(V_{\text {rot }} / c\right) \sqrt{1-\mu^{2}}$, where $\mu=\cos \theta$ (see Fig. 24a). It follows that the behavior of the line 
profile, in particular that of the DPS, depends on the emission power of the front part of the ring, and on the emission produced by the corresponding rear sector, which is left after being partially absorbed by the front part of the $\theta$-dependent, wavelength, displacement. A schematic representation of the line, emissionprofile is shown in Fig. 24c. In both scenarios, The actual widening of the DPS, depicted in Fig. 24b, or its apparent extent, shown in Fig. 24c, is accompanied, in both scenarios, by a reduction in the global, line-emission. The line shape depends in addition on the aspect angle at which the star+ring system is observed.

A more simple explanation of our difficulty to reproduce phase 3 may be due to our simple and crude, optically-thin, disk assumption for our 2D kinematical models. In most Be disks, the circumstellar gas becomes optically-thick in the inner dense parts of the disk, and the disk has an approximately-constant, surface-brightness in its optically-thick part (for a constant temperature disk). The peaks in the profile are related to the Keplerian velocities at the outer boundary between the optically thick and thin regions. We observe that the boundary between the optically thick and thin regions moves inwards, where the Keplerian velocities are faster, in a scenario such as that shown in Fig. 19, in which the disk density decreases proportionally everywhere inside the disk. This produces an increase in the DPS, as the emission decreases, as observed.

\section{Discussion and conclusion}

We have used the SIMECA code, based on a physical model as seen in Sect. 3, to interpret the VINCI data of KD, who used a simple model, which assumed that a 2D, elliptical, Gaussian, envelope superimposed on a uniform ellipse, describes the central distorted star. We find evidence of a polar wind with a 5\% contribution to the total flux and a spatial extension greater than $10 R_{\star}$, in agreement with the results obtained by KD. We conclude that at the time of the VINCI observations Achernar had either a small or no circumstellar disk, but it did have a polar, stellar wind with a poorly-constrained opening angle of approximately $\sim 20^{\circ}$.

The stellar and disk parameters used in this study are compatible with those obtained in Vinicius et al. (2006), that is they have the same photospheric density of $6.3 \times 10^{11}$ hydrogen atoms per $\mathrm{cm}^{3}$ and roughly the same effective temperature, i.e. $15000 \mathrm{~K}$ for Vinicius et al., whereas we adopted a latitudedependent, effective-temperature that varied from $8500 \mathrm{~K}$ (equator) to $20000 \mathrm{~K}$ (pole). The plot in Fig. 14, of Vinicius et al. (2006) showing the specific intensity at the equator as a function of the disk radial extension less than $3 R_{*}$, is also compatible with our finding of a wind+disk scenario with a disk less than $5 R_{*}$ since we lack interferometric data on small baselines to constrain strongly the disk size. more measurements for data of small baselines may not help because, if the disk is small, data of longer baselines is usually required to resolve its structure, or differentiate the disk and stellar photosphere visibilities. Since Achernar is the closest Be star, its photosphere is resolved on baselines of only a few tens of meters in the $K$-band. If we increased the baseline length, we would be able to study the flux ratio between the central star and its circumstellar disk $\left(F_{\star} /\left(F_{\text {env }}+F_{\star}\right)\right)$, or, on large baselines, to study limb or gravity darkening of the stellar photosphere in addition to the boundary between the star and the disk. As plotted in Fig. 2, we note that Achernar's disk is already resolved on small baselines. If we want to study its extension and geometry, we need to sample baselines of between 0 and $1.22 \lambda / D_{\text {env }}$, that is we need small baselines, as already justified in Sect. 4. Our results strongly disagree with the disk/star flux ratio of $27 \%$ in Vinicius et al. (2006), since we obtain a value $<5 \%$ at $2.2 \mu \mathrm{m}$. A value of $27 \%$ would have produced visibilities that were significantly different from the VINCI measurements.

The study of Achernar's spectroscopic data acquired between 1991 and 2002 completed by Vinicius et al. (2006), supported the presence of an evolving, equatorial disk. Thus, we used these $\mathrm{H} \alpha$ line-profiles to study their variations and found a clear signature of the formation-dissipation of the equatorial disk. The disk evolution follows 3 phases, the first two were reproduced with an outburst scenario, whereas the third phase, corresponding to the final contraction of the disk, may be difficult to reproduce due to the simple assumption of an optically-thin disk used in our 2D kinematical models. As already outlined in $\mathrm{KD}$, it appears that the polar wind may be present independently of the phase of the central star (B or Be phase), i.e. the polar, stellar-wind does not seem to be linked to the presence of a disk or a ring around the star.

Finally, we performed a $\chi^{2}$ fit to check whether the VLTI/VINCI visibility measurements of Achernar could be explained by a rotationally-deformed star (approximated by a uniform ellipse) and a binary companion, detected by Kervella \& Domiciano (2007), instead of a deformed star and a polar jet.

Since the number of free parameters is large and little is known about the companion, we fixed the uniform, ellipse parameters to be the values given by $\mathrm{KD}$, i.e. major axis = 2.13 mas, minor axis $=1.51$ mas, and major-axis, positionangle $=131.6^{\circ}$. The companion was approximated by a uniform disc with an angular diameter of 0.5 mas. The free parameters are the position of the companion (distance and position angle) and the flux ratio between the companion and Achernar. The models were calculated for a distance ranging from 3 to 300 mas with a step of 0.5 mas, and a position angle ranging from -180 to $180^{\circ}$ with a $1^{\circ}$ step. The flux ratio was varied from $1 \%$ to $10 \%$ in steps of $0.25 \%$. For a fixed flux ratio, there is no welldefined $\chi_{v}{ }^{2}$ minimum in the distance-position-angle plane that leads to a unique determination of the parameters. These local minima in the distance-position angle plane are lower for a flux ratio of $2.75 \%$, but in any case the reduced $\chi_{v}{ }^{2}$ is always greater than 1 , which is higher than the value found for the uniform ellipse plus polar-jet model $\left(\chi_{v}{ }^{2}=0.79 ; \mathrm{KD}\right)$. The $\chi_{v}{ }^{2}$ for the companion located at the position found by Kervella \& Domiciano de Souza (2007) is equal to 2.8 , and the flux ratio is $2.6 \%$. For this position and a fixed flux ratio of $1.8 \%$ (value from Kervella $\&$ Domiciano de Souza 2007), we obtain $\chi_{v}{ }^{2}=3$.

These results indicate that the VLTI/VINCI visibilities cannot be explained by a rotationally-distorted Be star and a companion alone, and the presence of a polar jet is indeed a necessary component to reproduce the observations. It is not excluded, however, that the companion could partially influence, the interferometric observations. As an attempt to quantify this influence we fitted the data with a 3-component model: uniform ellipse, polar jet, and companion. The reduced $\chi_{v}{ }^{2}$ obtained was almost identical to that measured by KD with the deformed-star-pluspolar-jet model, and the derived, flux-ratio between the companion and the central star is below $1 \%$. This indicates that the inclusion of the companion does not significantly improve the quality of the fit. Further observations would be required to constrain the physical parameters more accurately and the relative influence of the polar jet and of the companion.

Acknowledgements. The Programme National de Physique Stellaire (PNPS) and the Institut National en Sciences de l'Univers (INSU) with the Bonus Qualite Recherche (BQR) from the Observatoire de la Côte d'Azur are acknowledged 
for their financial supports. We would like to thank the referee Pr. Doug Gies for a careful reading of the paper and providing many helpful comments and suggestions. J.R.R. Tolkien is also acknowledged for helping us to find the right title for this paper.

\section{References}

Andrews, P. J., \& Breger, M. 1966, Observatory, 86, 108

Arias, M. L., Zorec, J., \& Frémat, Y. 2007, ASP CS, 361, 419

Balona, L. A., Engelbrecht, C. A., \& Marang, F. 1987, MNRAS, 227, 123

Carciofi, A. C., Domiciano de Souza, A., Magalhes, A. M., Bjorkman, J. E., \& Vakili, F. 2008, ApJ, in press [arXiv:0801.4901v1]

Castelli, F., \& Kurucz, R. L. 2003, IAU Symp., 210, 20

Castor, J. I., Abbott, D. C., \& Klein, R. I. 1975, ApJ, 195, 157

Chauville, J., Zorec, J., Ballereau, D., et al. 2001, A\&A, 378, 861

Cidale, L., \& Ringuelet, A. 1989, PASP, 101, 417

Claret, A. 1998a, A\&A, 335, 647

Claret, A. 1998b, A\&AS, 131, 395

Dachs, J., Maitzen H. M., Moffat, A. F. J., Sherwood, W. A., \& Stift, M. 1977, A\&A, 56, 417

Dachs, J., Eichendorf, W., Schkeicher, H., et al. 1981, A\&AS, 43, 427

Dachs, J., Hanuschik, R. W., Kaiser, D., et al. 1986, A\&AS, 63, 87

Dachs, J., Hummel, W., \& Hanuschik, R. W. 1992, A\&AS, 95, 437

Domiciano de Souza, A., Kervella, P., Jankov, S., et al. 2003, A\&A, 407, L47

Fremat, Y., Zorec, J., Hubert, A. M., \& Floquet, M. 2005, A\&A, 440, 305

Gies, D. R., McKibben, W. P., Kelton, P. W., Opal, C. B., \& Sawyer, S. 1990, AJ, 100, 1601

Gies, D. R., Bagnuolo Jr., W. G., Baines, E. K., et al. 2007, ApJ, 654, 527

Hanuschik, R. W., Kozok, J. R., \& Kaiser, D. 1988, A\&A, 189, 147

Hanuschik, R. W., Hummel, W., Sutorius, E., et al. 1996, A\&AS, 116, 309
Hubeny, I. 1988, Computer Physics Comm., 52, 103

Hubeny, I., \& Lanz, T. 1995, ApJ, 439, 875

Jaschek, C., Jachek, M., \& Kucewicz, B. 1964, Z. Astrophys., 59, 108

Kervella, P., \& Domiciano de Souza, A. 2006, A\&A, 453, 1059 (KD)

Kervella, P., \& Domiciano de Souza, A. 2007, A\&A, 474, L49

Kurucz, R. L. 1993, CD-ROM No. 13, Cambridge, Mass., SAO

Lamers, H. J. G. L. M., \& Pauldrach, A. W. A. 1991, A\&A, 244, L5

Mc David, D. 2005, in The Nature and Evolution of Disks around Hot Stars,

ed. R. Ignace, \& K. G. Gayley (San Francisco: ASP), ASP Conf. Ser., 337, 265

Meilland, A., Stee, Ph., Zorec, J., \& Kanaan, S. 2006, A\&A, 455, 953

Meilland, A., Stee, Ph., Vannier, M., et al. 2007, A\&A, 464, 59

Owocki, S. P. 2004, in Stellar Rotation, ed. A. Maeder, \& P. Eenens (San Francisco: ASP), Proc. IAU Symp., 215, 515

Porter, J. M., \& Rivinius, T. 2003, PASP, 115, 1153

Quirrenbach, A., Bjorkman, K. S., Bjorkman, J. E., et al. 1997, ApJ, 479, 477

Slettebak, A. 1982, ApJ, 50, 55

Stee, Ph. 2003, A\&A, 403, 1023

Stee, Ph., \& Araújo, F. X. 1994, A\&A, 292, 221

Stoeckley, T. R. 1968, MNRAS, 140, 141

Towsend, R. H. D., Owocki, S. P., \& Howard, I. D. 2004, MNRAS, 350, 189

Tycner, Ch. 2004, Ph.D. Thesis, University of Toronto

Tycner, Ch., Gilbreath, G. C., Zavala, R. T., et al. 2006, AJ, 131, 2710

Vinicius, M. M. F., Zorec, J., Leister, N. V., \& Levenhagen, R. S. 2006, A\&A, 446,643

von Zeipel, H. 1924, MNRAS, 84, 665

Yudin, R. V. 2001, A\&A, 368, 912

Zamanov, R. K., Reig, P., Marti, J., et al. 2001, A\&A, 367, 884

Zorec, J., Mochkovitch, R., \& Divan, L. 1988, C.R. Acad. Sci. Paris, 306, 1265

Zorec, J., Frémat, Y., \& Domiciano de Souza, A. 2007, in Active OB Stars: Laboratories for Stellar and Circumstellar Physics, ASPC, 361, 542 